\title{
A BASIS FOR PRONOMINAL ANAPHORA RESOLUTION USING A MODEL OF WORKING MEMORY AND LONG-TERM MEMORY
}

\author{
Clifford James Thompson \\ B.Sc., University Of Northern British Columbia, 2001
}

Thesis Submitted In Partial Fulfillment Of

The Requirements For The Degree Of

Master Of Science

In

Mathematical, Computer, And Physical Sciences

(Computer Science)

The University Of Northern British Columbia

(C) Clifford James Thompson, 2006

May 2006 


\begin{abstract}
This thesis presents a new theory of information modelling in natural language processing that attempts to resolve anaphoric references, while also addressing the problem of knowledge complexity. A modular model of semantic representation is introduced that addresses the deficiencies of existing representations, as well as the drawbacks associated with expanding these semantic representations. Rather than using a single semantic representation to model human knowledge and the knowledge within a sentence, the theory proposes a modular, multi-level model which is based around a semantic network. The behaviour of the model uses theories on the nature of working and long-term memory from cognitive psychology. Two methods of artificial neuron activation and decay were implemented - the ACT-R model and the Thompson model. Maximum success rates of $54.10 \%$ and $83.61 \%$ were achieved for The Three Brothers corpus, and maximum success rates of $56.00 \%$ and $86.67 \%$ were achieved for the Rumpelstiltskin corpus.
\end{abstract}


This thesis is approved

for recommendation to the

Graduate Council.

Thesis Director:

Dr. Charles Brown

\section{Thesis Committee:}

Dr Charles Brown

Dr. William Owen

Dr. Liang Chen 


\section{Table of Contents}

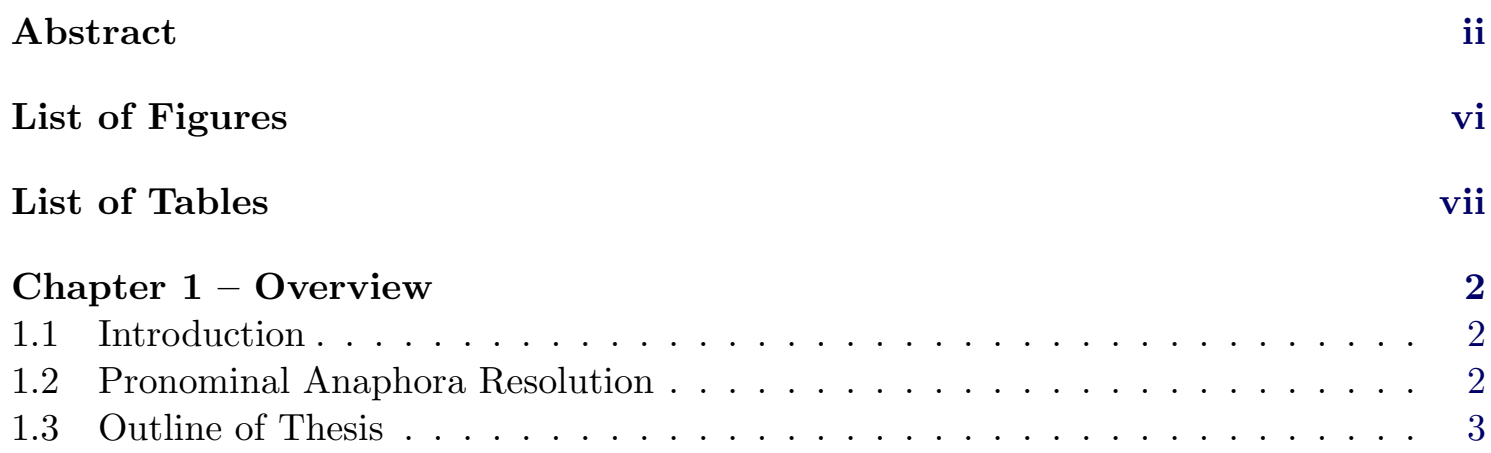

Chapter 2 - Declarative Semantic Structures 5

2.1 Semantic Networks . . . . . . . . . . . . . . . . . . . . . 6

2.2 Conceptual Graphs . . . . . . . . . . . . . . . . . . . . . 7

2.3 Conceptual Hierarchies . . . . . . . . . . . . . . . . . . . . . . . . . 9 9

2.4 Event Timeline Models . . . . . . . . . . . . . . . . . . . . . . . . 10

2.5 Thematic Roles . . . . . . . . . . . . . . . . . . . . . . 11

2.6 Logical Form and Quasi Logical Form . . . . . . . . . . . . . . . . . . . 13

2.7 Conclusion . . . . . . . . . . . . . . . . . 16

$\begin{array}{lr}\text { Chapter } 3 \text { - Working \& Long-Term Memory } & 17\end{array}$

3.1 Working Memory Models . . . . . . . . . . . . . . . . . 17

3.2 Long-Term Memory Models and Associativity . . . . . . . . . . . . . . . . . 19

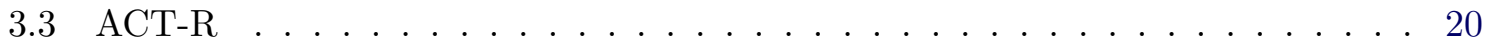

3.4 Computational Models of Memory . . . . . . . . . . . . . . . . . . 22

3.5 Neural Network Models of Associative Memory . . . . . . . . . . . . . . . . . 23

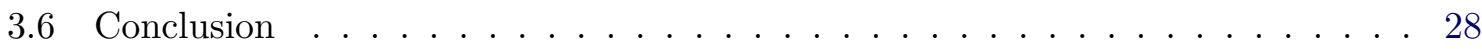

Chapter 4 - Theory on Combining Semantic Structures 29

4.1 Linking Semantic Structures . . . . . . . . . . . . . . . . . . . . . . . 29

4.2 Modularization and The Human Mind . . . . . . . . . . . . . . . . . 32

4.3 Conclusion . . . . . . . . . . . . . . . . . . . . 32

Chapter 5 - Anaphoric Reference and Reference Resolution 33

5.1 Types of Anaphoric Reference . . . . . . . . . . . . . . . . . . . . . 33

5.2 Problems in Anaphora Resolution . . . . . . . . . . . . . . . . 33

5.3 Anaphora Resolution Algorithms . . . . . . . . . . . . . . . . . . . . . . . . . . . . . . . .

5.4 Resolution Failure . . . . . . . . . . . . . . . . . . . 37

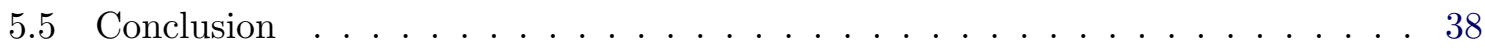


Chapter 6-System Modelling $\quad 39$

6.1 Modelling Long-Term Memory . . . . . . . . . . . . . . . . . . . . . . . . . . 39

6.2 Working Memory . . . . . . . . . . . . . . . . . . . 41

6.3 Prolog Model of Working Memory . . . . . . . . . . . . . . . . . . . 42

6.4 English Grammar Rules . . . . . . . . . . . . . . . . . . . . . . . 43

6.5 Annotated Parse Tree Model . . . . . . . . . . . . . . . . . . . . . . 44

6.6 Lexical Feature Sets . . . . . . . . . . . . . . . . . . . . . 45

6.7 Anaphora Resolution Algorithm . . . . . . . . . . . . . . . . . . 46

6.8 Summary of Chapter . . . . . . . . . . . . . . . . . . . 47

Chapter 7 - System Testing $\quad 49$

7.1 Overview of Testing Methodology . . . . . . . . . . . . . . . . 49

7.2 Testing Platform . . . . . . . . . . . . . . . . . . . . 49

7.3 Decay Rates. . . . . . . . . . . . . . . . . . . . 49

7.4 Default Memory Configuration . . . . . . . . . . . . . . . 50

7.5 Testing Procedure . . . . . . . . . . . . . . . . . . . 50

7.6 Anaphora Resolution Results . . . . . . . . . . . . . . . . . . 51

7.7 Working Memory Capacity Results . . . . . . . . . . . . . . . 52

7.8 Discussion of Results . . . . . . . . . . . . . . . . . . . . 52

7.9 Classification of Observed Error Types . . . . . . . . . . . . . . 54

Chapter 8 - Conclusions and Future Work 59

8.1 Comparison with Related Work . . . . . . . . . . . . . . . . 59

8.2 Future Work . . . . . . . . . . . . . . . . . . . 60

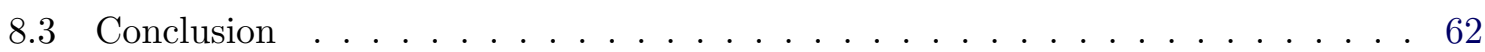

$\begin{array}{ll}\text { Bibliography } & 63\end{array}$

$\begin{array}{ll}\text { Appendix A - Corpora } & 71\end{array}$

$\begin{array}{ll}\text { Appendix B - Tagged Corpora } & 75\end{array}$ 


\section{List of Figures}

2.1 Probabilistic Semantic Network . . . . . . . . . . . . . . . . . . 7

2.2 Conceptual Relations of Arity (a) 1 (b) 2 and (c) $3 \ldots \ldots \ldots$

2.3 Conceptual Graph for Mary gave John the book. . . . . . . . . . . . . . . . . 8

2.4 Conceptual Graph for John is going to Boston . . . . . . . . . . . . . . . . . 8

2.5 Another Conceptual Graph for John is going to Boston . . . . . . . . . . . . . 9

2.6 Conceptual Hierarchy . . . . . . . . . . . . . . . . . . . . . . . . 9 9

2.7 Timeline Model . . . . . . . . . . . . . . . . . . . . . 10

2.8 Reichenbach Timeline Model . . . . . . . . . . . . . . . . . . 12

2.9 [Altmann 1999] Thematic Implausibility: . . . . . . . . . . . . . . . . . . 13

2.10 Sentences Implying The Same Event . . . . . . . . . . . . . . . . . . . . 13

2.11 Logical Form Rules from the Core Language Engine [Alshawi et al 1989] . . . . 15

2.12 Logical Form for Every doctor visited Mary . . . . . . . . . . . . . . . . . . . . . 15

3.1 Baddeley and Hitch's Working Memory Model . . . . . . . . . . . . . . . . 19

3.2 Hunt's Distributed Memory Model . . . . . . . . . . . . . . . . . . . . . 24

3.3 Biological Neuron . . . . . . . . . . . . . . . . . . . . . 25

3.4 Multi-Input Neuron . . . . . . . . . . . . . . . . . . . 26

3.5 Multi-Layered Artificial Neural Network . . . . . . . . . . . . . . . . . 27

4.1 Semantic Network Linking Semantic Representations . . . . . . . . . . . . . . . . 30

4.2 Multiple Semantic Representations . . . . . . . . . . . . . . . . . . . . 31

5.1 Brown's Anaphora Resolution Algorithm . . . . . . . . . . . . . . 36

5.2 Two Parsings of I saw a man with a telescope . . . . . . . . . . . . . . . . 37

5.3 Mitkov's Anaphora Resolution Algorithm . . . . . . . . . . . . . . . . . . 38

6.1 Annotated Parse Trees with (a) Unresolved and (b) Resolved Antecedents . . . 45

6.2 Pronoun Reference Resolution Algorithm Pseudo-code . . . . . . . . . . . . . . 47

7.1 The ACT-R Model Resolution Results - 3 Brothers . . . . . . . . . . . . . . . . 51

7.2 The ACT-R Model Resolution Results - Rumpelstiltskin . . . . . . . . . . . . . 52

7.3 The Thompson Model Resolution Results - 3 Brothers . . . . . . . . . . . . . . 53

7.4 The Thompson Model Resolution Results - Rumpelstiltskin . . . . . . . . . . . 54

7.5 The ACT-R Model Working Memory Max Capacity - 3 Brothers . . . . . . . . 55

7.6 The ACT-R Model Working Memory Max Capacity - Rumpelstiltskin . . . . . 56

7.7 The Thompson Model Working Memory Max Capacity - 3 Brothers . . . . . . 56

7.8 The Thompson Model Working Memory Max Capacity - Rumpelstiltskin . . . 57

7.9 Working Memory Contents Comparison - 3 Brothers . . . . . . . . . . . . . 57

7.10 Working Memory Contents Comparison - Rumpelstiltskin . . . . . . . . . . . 58 


\section{List of Tables}

2.1 Example Sentences Taken from $[$ Allen 1995] . . . . . . . . . . . . . . . 11

2.2 Thematic Roles . . . . . . . . . . . . . . . . . . . . . . . . . . 14

6.1 Semantic Network Predicates . . . . . . . . . . . . . . . . . . . 40

6.2 Lexical Feature Set . . . . . . . . . . . . . . . . . . . . 46

6.3 Semantic Feature Set . . . . . . . . . . . . . . . . . . 46

8.1 Algorithm Comparison . . . . . . . . . . . . . . . . . 60 


\section{Acknowledgements}

This thesis would not have been a success without the patience and hard work of many individuals to whom I am greatly indebted. First, and foremost, I must thank my supervising professor, Dr. Charles Brown, for introducing me to the field of Artificial Intelligence and encouraging me to enter graduate studies. Your selfless dedication of time and effort will always be remembered. I must also thank Dr. William Owen for his advice in a field that was completely foreign to me. Your help and direction allowed me to achieve the multi-disciplinary approach that I persevered for. I also would like to thank Dr. Liang Chen for his advice and comments, Dr. Paul Siakaluk for taking the time to be the external examiner for my thesis, Erin Beveridge for the original $\mathrm{LAT}_{\mathrm{E} X} \mathrm{X}$ style files, and UNBC for awarding me the Graduate Entrance Scholarship (which made the decision of graduate studies much easier). And finally, I must thank my wife Coral. Without your

love, advice, encouragement, and general kick in the butt, this thesis would have not have been possible. 


\section{CHAPTER ONE}

\section{Overview}

She had a pretty gift for quotation, which is a serviceable substitute for wit.

- W. Somerset Maugham

\section{$1.1 \quad$ Introduction}

It seems ironic that although natural languages are very difficult to model, the languages themselves are quite effective and efficient for communication. If humans use their native language with ease, then why is it so hard for computers to understand natural languages? One of the most obvious answers is that the human brain is so complex. The complexity of human knowledge, and the medium on which it is stored and processed, cannot be understated.

This thesis presents a new theory of memory modelling in natural language processing that attempts to resolve anaphoric references, while also addressing the problem of complexity. Rather than using a single semantic model to represent human knowledge and the knowledge within a sentence, the theory proposes a more general model where multiple semantic representations can be used in a system that models the observed behaviour of working and long-term memory.

\subsection{Pronominal Anaphora Resolution}

The goal of this thesis was to develop a multi-level model of human memory that is modular and flexible, processes multiple well-known semantic representations such as semantic networks, conceptual graphs and quasi-logical form, and uses these models to resolve 
anaphoric references. In-particular, this thesis focused on the resolution of simple pronouns such as he, she, they, etc. In the most general of cases, pronoun resolution is quite simply a matter of searching backwards through a corpus of text until the first noun phrase that matches such attributes as number and gender is found:

Bright and early the next morning, the shoemaker 1 rose and went to his ${ }_{1}$ work bench. To his amazement, there on the table were two shoes ${ }_{2}$, already finished. They 2 were beautifully made, neat and true, and with not a single false stitch.

The situation can be made slightly more complex by making antecedents separate entities in the context:

For some time that same thing happened, until the good man $_{1}$ and his wife ${ }_{2}{ }_{3}$ were thriving and prosperous. But they ${ }_{3}$ were not satisfied to have so much done for them ${ }_{3}$ and not know to whom they 3 should be grateful.

But of course, this is not always the case. The next example, adapted from [Sidner 1983], demonstrates where this method of resolution can break down:

My neighbours $_{1}$ have a monster Harley $12000_{2}$. They 3 are really huge but gas efficient bikes.

In the second sentence, if an individual was to read just the pronoun they, their initial preference for the reference may not be a monster Harley 1200 based on number alone. In this context, a common preference for the pronoun they would be my neighbours. After reading the remainder of the second sentence, it is apparent that this conclusion was incorrect. Given the additional context, common knowledge concludes that the neighbours are not motorcycles ${ }^{1}$.

\subsection{Outline of Thesis}

Chapter 2 discusses numerous semantic representations that have been introduced over the past few decades. The general domain of use is covered for each semantic representation

\footnotetext{
${ }^{1}$ That is unless your neighbours actually are motorcycles.
} 
as well as some of the drawbacks for each structure. Chapter 3 will discuss the evolution of theories of short-term and long-term memory as well as current theories in human memory. Several modern psychological models of short-term and long-term memory will be elaborated on, as well as some computational memory models. In Chapter 4, the discussion on semantic representations will move towards a theory on combining semantic representations to overcome their individual deficiencies with the intention of creating a system that is easier to understand and easier to expand. The theory will also model human behaviour more closely.

Chapter 5 will discuss the current state of understanding in pronominal anaphoric reference and anaphora resolution. Several theoretical problems will be introduced and discussed. A number of modern anaphora resolution algorithms will be presented that attempt to solve anaphoric reference issues. The chapter will conclude with an examination of what occurs when humans fail to resolve anaphoric references. In Chapter 6, the combined semantic representation model, memory models, and anaphora resolution algorithms will be integrated into a system that will attempt to solve anaphoric reference problems introduced in Chapter 5. Chapters 7 will cover the methodology for testing the implemented model, the results of testing, and a discussion of those results. Chapter 8 will conclude the thesis by comparing the testing results with the results of other models, and a discussion on how the model presented in this thesis could be improved. 


\title{
CHAPTER TWO
}

\section{Declarative Semantic Structures}

\author{
Oh, and sir, you're wrong. We won't be apart - we just won't be together. \\ - Arnold J. Rimmer (Holoship)
}

Although many types of semantic representations have emerged during the history of natural language processing research, understanding in the domain of semantics is still limited. Some models fall short and are intended for a limited knowledge domain. Others can be expanded but the resulting expansions are often unclear or more difficult to computationally manage.

In this chapter, a number of semantic representation models are examined. As each model is investigated, the shortcomings of each model will be shown. The examination of these shortcomings will lay the initial groundwork for a hypothesis on improving these models. By integrating each semantic model separately into a larger, multi-level system, it is hypothesized that the resulting system would be easier to expand than a system with a single complex semantic model, and would provide a diverse knowledge base from which an anaphora resolution algorithm, or group of algorithms, could draw from. James Allen makes a statement in [Allen 1995] to this vain:

...a vigorous debate about knowledge representation is actually the result of each of the debaters focusing on one of the aspects of representation without considering the concerns of the other.

Humans apply much implicit knowledge when understanding an utterance. Information in long-term memory is not considered in many structures, and even if it is, the information is stored only at the discourse level. Ignoring the complexity of a human knowledge base only trivializes the vast learning power of the human mind. 


\subsection{Semantic Networks}

Some of the earliest research with respect to semantic networks can be found in [Quillian 1968] and [Collins and Quillian 1969]. Semantic networks were first introduced as a model of human memory. How semantic networks are realized as models in computation is quite a broad topic. Interpretation varies from graphs with concepts as nodes and the associations between the nodes as links, to more complex graphs such as Sowa's conceptual graphs [Sowa 1984] or conceptual hierarchies [Ma and Isahara 2000] [Chung and Moldovan 1993] . For the purposes of this thesis, semantic networks will be restricted to the first definition, graphs with concepts as nodes and associations as links.

Figure 2.1 represents a semantic network that has a strength associated with each link. The network roughly represents an artificial neural network, which will be discussed further in Chapter 3. Each node ${ }^{1}$ represents a single topic or concept. Thus, the relationship between two semantic concepts is based on the strength of the association between the two semantic concepts. The drawback of this model is that it only models a loose relationship between topics. It does not identify what the relationship is. The next example illustrates how drawing the appropriate knowledge from a semantic network would be difficult:

John had a son named Bob. His son is an excellent skier.

In this example, an anaphora resolution algorithm would have a difficult time resolving the possessive pronoun His without the father-son relationship being modelled more explicitly.

[Kazuhiro et al 1992] and [Berger et al 2004, Belew 1987] demonstrate examples of semantic networks with weighted links being used in kana-kanji conversion and information retrieval, respectively. In Chapter 4 we will see that semantic networks will not be used to model knowledge directly. Rather, they will be used to connect semantic concepts and their associated semantic representations.

\footnotetext{
${ }^{1}$ A node does not necessarily represent a single neuron within the human brain. A node could represent a group of neurons.
} 


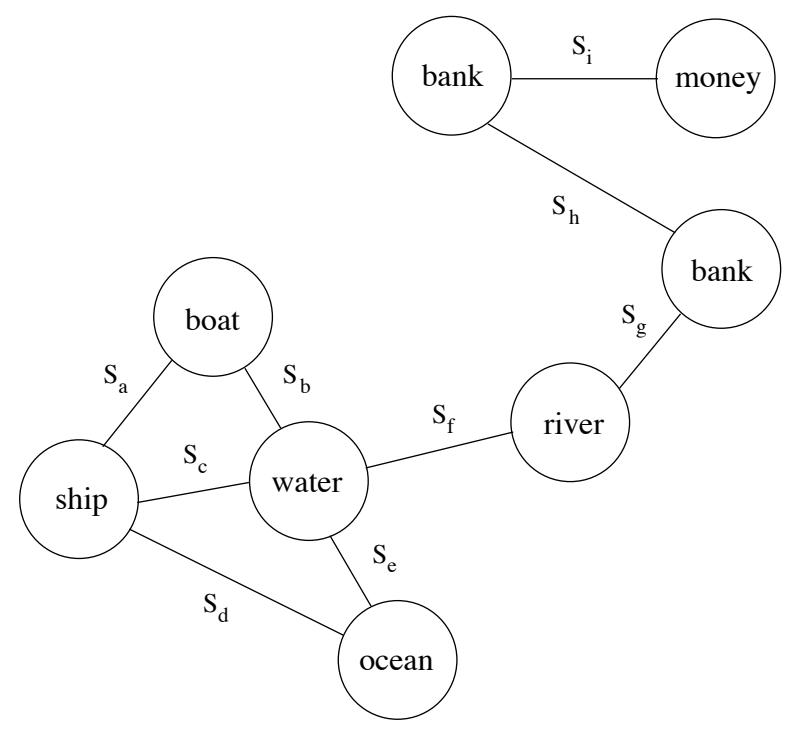

Figure 2.1: Probabilistic Semantic Network

\subsection{Conceptual Graphs}

Some of the earliest work with respect to conceptual graphs can be attributed to Sowa in [Sowa 1976, Sowa 1979, Sowa 1984]. Conceptual graphs are defined as a directed bipartite graph with two types of nodes. Each node in the graph can be either a concept or a conceptual relation. Concepts can be concrete (such as cat), or they can be abstract (such as sadness). Conceptual relations can have an arity of $n \geq 1$. Figure 2.2 illustrates conceptual relations with various arities.

Conceptual graphs are not limited to the simple relations shown in Figure 2.2. They can also model simple sentences, as seen in Figure 2.3. Since conceptual graphs are used extensively in database systems, relational database theory allows us to perform certain operations to obtain new conceptual graphs, such as copy, restrict, join, and simplify. In their basic form, conceptual graphs do not model the strength of relationships. Common knowledge dictates that information stored in long-term memory is not as concrete as the conceptual graph model it to be. Fuzziness with respect to relations is not accounted for. Conceptual graphs, as defined by Sowa, do not model temporal information implied by verb tense and verb aspect. This deficiency is apparent in Figure 2.4 adapted from 


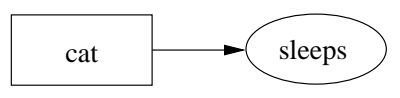

(a)

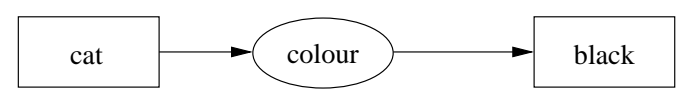

(b)

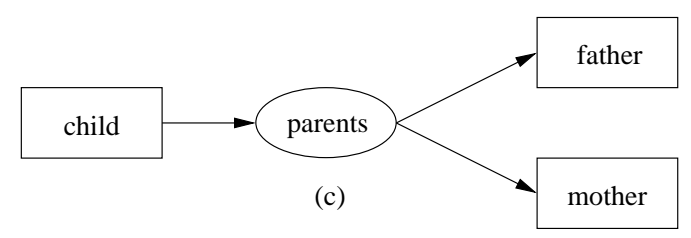

Figure 2.2: Conceptual Relations of Arity (a) 1 (b) 2 and (c) 3

[Sowa 2000]

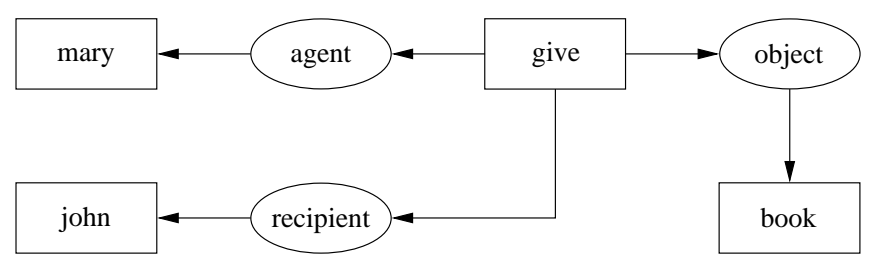

Figure 2.3: Conceptual Graph for Mary gave John the book.

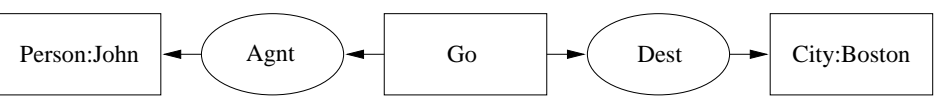

Figure 2.4: Conceptual Graph for John is going to Boston

It is apparent that the tense and aspect have been lost for the verb phrase is going. Tense and aspect could be reflected by adding another relation, as shown in Figure 2.5.

How would we model one event occurring before another event? Another relation would seem to be a possible answer. This process of adding relations to reflect previous missed information could lead to quite a rat's nest. It becomes difficult to separate verbs and nouns from other semantic information without adding more relations, and it potentially becomes a model that is more difficult to expand and maintain. 


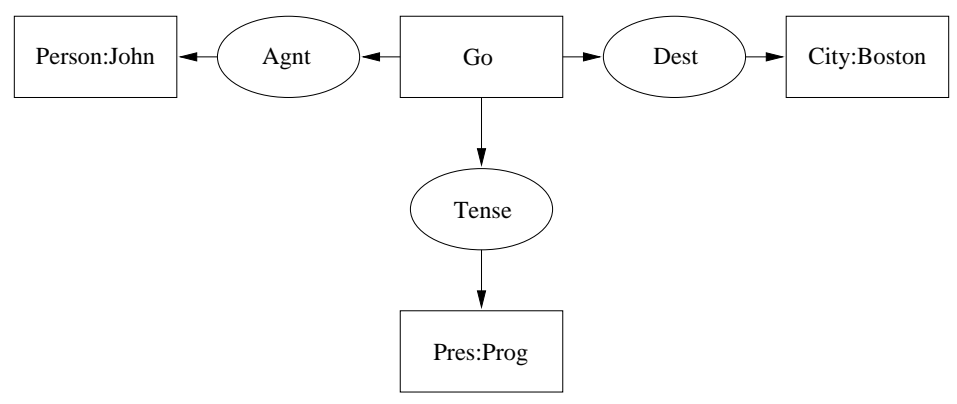

Figure 2.5: Another Conceptual Graph for John is going to Boston

\subsection{Conceptual Hierarchies}

Conceptual hierarchies are a very common structure in object oriented programming. They allow programmers to show how certain objects inherit the properties of another object. Figure 2.6 illustrates an example of how classification and sub-classification have been observed by biology throughout the world. Subclasses inherit attributes from their superclass as well as adding their own attributes. Research in cognitive categorization, such as [Kay 1971, Rosch et al 1976], suggests that the human mind stores and groups information based on taxonomy in long-term memory. One of the main advantages of conceptual hierarchies is that they are very efficient at storing information [Ma and Isahara 2000]. Information common to many concepts is only stored once.

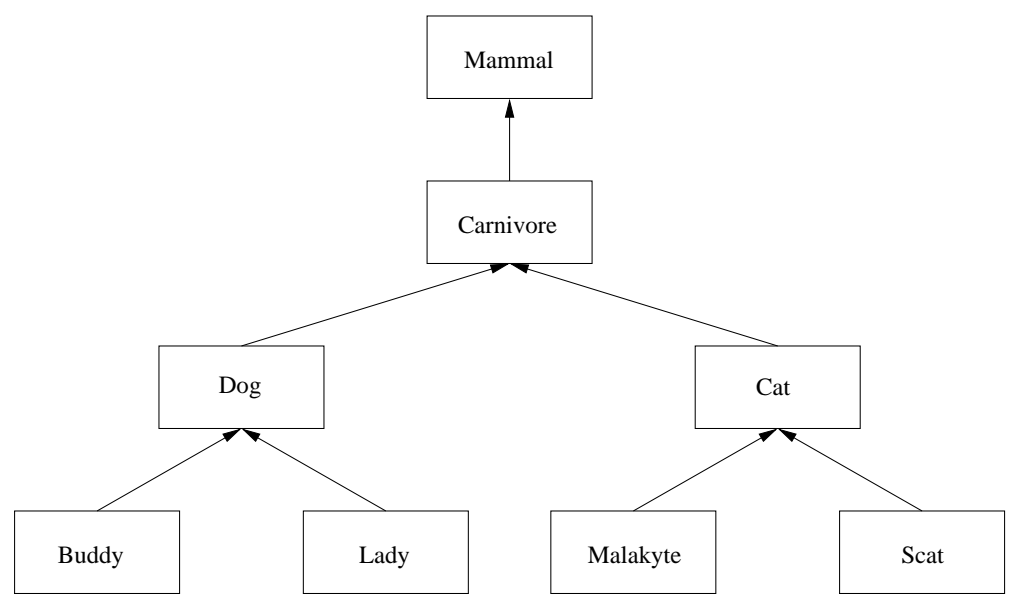

Figure 2.6: Conceptual Hierarchy 
One the main disadvantages of conceptual hierarchies is their intended use: modelling concepts and the inheritance of characteristics between concepts. They are not intended to model entire sentences or any kind of temporal information.

\subsection{Event Timeline Models}

\section{The Bull Framework}

In [Culce-Murcia and Larsen-Freeman 1999], Celce-Murcia and Larsen-Freeman adapted the The Bull Framework [Bull 1960] (originally created for Spanish) for teaching ESL students English. The Bull Framework proposes four axes of time: past, present future, and hypothetical. The first three axes contain a point of reference in the centre and the times occurring before and after the time of reference to the left and right, respectively. Figure 2.7 demonstrates an example using the verb ski. The fourth axis, hypothetical, is used to model hypothetical events, for example, events created using constructions like if..then.

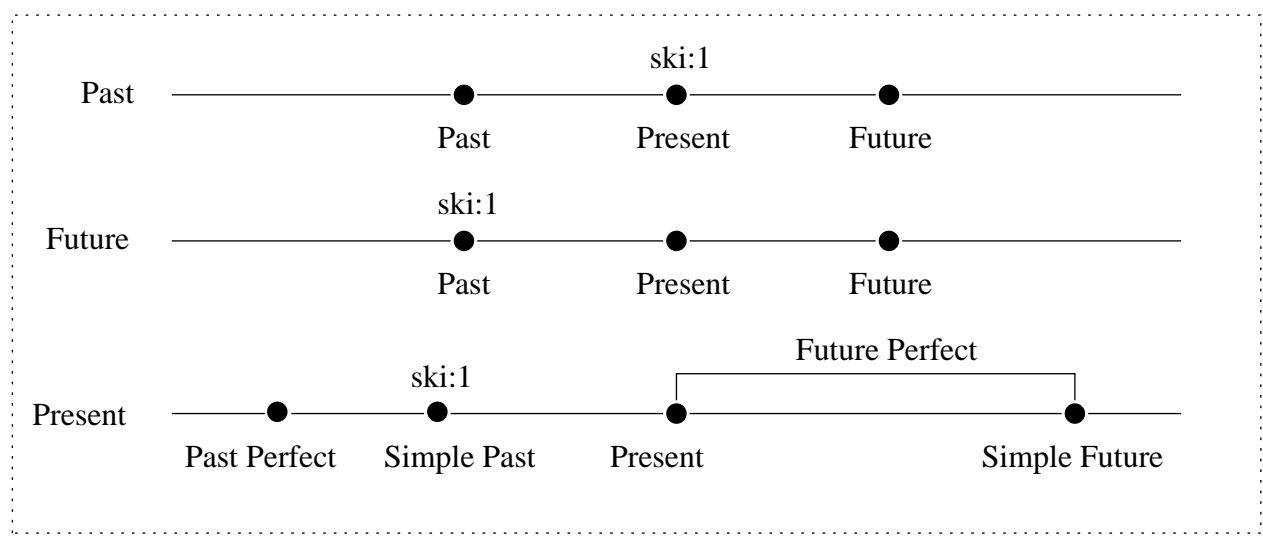

"I skied in the Whistler-Blackcomb backcountry."

Figure 2.7: Timeline Model

Since the Bull Framework was only intended for explaining verb tenses, it does not suitably model many aspects of natural languages such as nouns, adjectives, and adverbs. 


\section{The Reichenbach Theory}

In [Allen 1995], Allen presents Reichenbach's theory on timeline representation from [Reichenbach 1947]. Reichenbach theorized that each verb embeds information about three points in time: time of speech $(\mathrm{S})$, time of the event/state(E), and time of reference $(\mathrm{R})$. In the simple aspect ${ }^{2}$, the time of the event/state and the time of reference are always equivalent. This equivalency does not exist for the perfect and posterior aspects ${ }^{3}$. Figure 2.8 gives a table outlining the various timelines for different tense and aspect combinations. Table 2.1 shows some example sentences and their tense/aspect. Reichenbach methodology is very similar to that of Bull's in that they both attempt to model a type of temporal ordering, which is implied by varying tense and aspect combinations in sentences. Naturally, Reichenbach's theory does not attempt to account for the nature of nouns, adjectives, and adverbs.

\begin{tabular}{|c|c|}
\hline Tense & Example Sentence \\
\hline \hline Simple Present & Jack sings \\
\hline Simple Past & Jack sang \\
\hline Simple Future & Jack will sing \\
\hline Perfect Present & Jack has sung \\
\hline Perfect Past & Jack had sung \\
\hline Perfect Future & Jack will have sung \\
\hline Posterior Present & Jack is going to sing \\
\hline Posterior Past & Jack was going to sing \\
\hline Posterior Future & Jack will be going sing \\
\hline
\end{tabular}

Table 2.1: Example Sentences Taken from [Allen 1995]

\subsection{Thematic Roles}

Thematic roles are linguistic entities (embodied in the form of noun phrases) that satisfy certain semantic constraints implied by the main verb phrase of a sentence. The idea of

\footnotetext{
${ }^{2}$ It must be noted that the progressive aspect and perfect progressive aspect are missing from Allen's listings.

${ }^{3}$ Allen refers to simple as being a tense, not an aspect.
} 


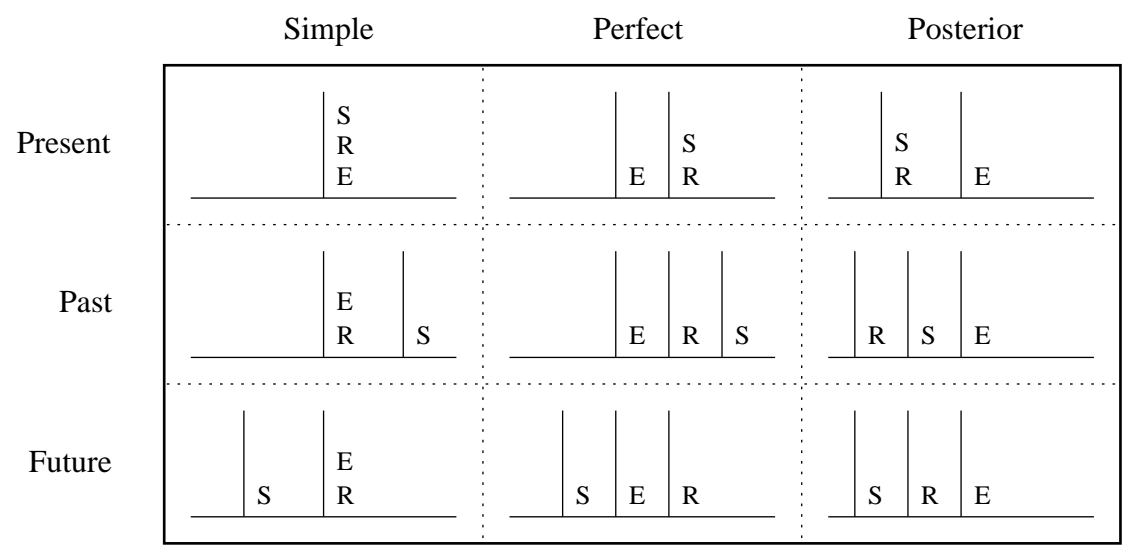

Figure 2.8: Reichenbach Timeline Model

thematic roles draws a close parallel to the morphological case systems found in languages such as German and Latin, but expands on the case system by adding a much larger number of cases.

Verb phrases require that these thematic roles are present before a sentence can make sense semantically. Altmann demonstrated in [Altmann 1999] that even if all thematic roles are met for a verb phrase, if the antecedent of a thematic role is not plausible, the sentence will not make sense. Figure 2.9 gives an example of (a) an implausible antecedent to a thematic role, and (b) a plausible antecedent to a thematic role. A major drawback of the Thematic Role model is that it only considers concepts at the sentence level. It does not attempt to address how concepts can be inter-related throughout are large body of text.

The structure of English allows thematic roles to be located at different syntactic positions within a sentence. The result is a sentence with a different syntactic structure and more emphasis can be placed on certain roles. Although the syntactic structure is different, when constructed properly, the new sentence should describe the same event. Figure 2.10 gives an example. It is apparent that the antecedents of thematic roles can be extracted from a sentence based on their semantic contribution to that sentence, rather than the syntactic contribution. Table 2.2 outlines some of the thematic roles proposed by Sowa in [Sowa 2000]. 
(a) A young toddler was running around his playroom. It was empty except for some chairs in one corner and some pet cats in the other. He chased a chair that he had run into before.

(b) A young toddler was running around his playroom. It was empty except for some chairs in one corner and some pet cats in the other. He bumped a chair that he had run into before.

Figure 2.9: [Altmann 1999] Thematic Implausibility:

(a) Bart threw a chicken at the house.

(b) A chicken was thrown at the house by Bart.

Figure 2.10: Sentences Implying The Same Event

\subsection{Logical Form and Quasi Logical Form}

The Core Language Engine was developed at the Stanford Research Institute and The Center for the Study of Language and Information at Stanford University. The methods of anaphora resolution in the Core Language Engine [Alshawi et al 1989] are heavily motivated by its internal semantic representation, logical form and quasi-logical form. Quasi-logical form is based on first order logic, which has been used widely in the fields of philosophy and linguistics. The structure of logical forms is motivated by the desire to use and extend first order logic, which is well suited for modelling quantifier scoping and anaphora.

This section will discuss the Core Language Engine's fully scoped logical form, and a logical form where scoping rules are relaxed for reference resolution, quasi-logical form. Although logical form and quasi-logical form were designed to handle many types of reference phenomena such as unscoped quantifiers, unscoped descriptions, and unresolved relations, the phenomenon that will be focused on is unresolved reference. Resolution in 


\begin{tabular}{|c|}
\hline Agent \\
\hline Beneficiary \\
\hline Completion \\
\hline Destination \\
\hline Duration \\
\hline Effector \\
\hline Experiencer \\
\hline Instrument \\
\hline Location \\
\hline Matter \\
\hline Medium \\
\hline Origin \\
\hline Path \\
\hline Patient \\
\hline Point In Time \\
\hline Recipient \\
\hline Result \\
\hline Start \\
\hline Theme \\
\hline
\end{tabular}

Table 2.2: Thematic Roles

the Core Language Engine uses a set of reference resolution rules that propose possible logical forms that can transform a quasi-logical form statement into a logical form statement. Fully resolved logical forms must conform to the following set of properties:

- should be expressions in a disambiguated language.

- should be suitable for representing the meanings of natural language expressions.

- should provide a suitable medium for the representation of knowledge expressed in natural language, and they should be a suitable vehicle for reasoning.

Figure 2.11 lists some of the grammar rules used in the Core Language Engine to model the logical form language, and Figure 2.12 shows an example of logical form for the sentence Every doctor visited Mary.

Not all references can be resolved immediately using logical form. Sentences such as (1) Most doctors read every article, and (2) the bishops arrived contain references where the scope of quantification is not exactly clear. In sentence (1), does each doctor in 


$$
\begin{aligned}
& \left\langle l f_{-} \text {formula }\right\rangle \rightarrow \text { quant }(\langle q \text { quantifier }\rangle,\langle\text { variable }\rangle,\langle\text { restriction }\rangle,\langle\text { body }\rangle) \\
& \left\langle\text { lf } _ { - } \text { formula } \rightarrow \left[\left\langle\text { functor },\left\langle\text { argument }_{1}\right\rangle,\left\langle\text { argument }_{2}\right\rangle, \cdots,\left\langle\text { argument }_{n}\right\rangle\right]\right.\right. \\
& \langle\text { functor }\rangle \rightarrow\langle\text { atom }\rangle \\
& \langle\text { quantifier }\rangle \rightarrow \text { forall } \mid \text { exists } \mid \cdots \\
& \langle\text { restriction }\rangle \rightarrow\left\langle l f_{-} \text {formula }\right\rangle \\
& \langle\text { body }\rangle \rightarrow\left\langle l f_{-} \text {formula }\right\rangle \\
& \langle\text { argument }\rangle \rightarrow\left\langle l f_{-} \text {formula }\right\rangle
\end{aligned}
$$

Figure 2.11: Logical Form Rules from the Core Language Engine [Alshawi et al 1989] quant (forall, D, [doctor $1, \mathrm{D}]$,

[past, quant (exists, E, [event, E] , [visit,E,D,mary1])])

Figure 2.12: Logical Form for Every doctor visited Mary

the most doctors set read all articles or does the most doctors set collectively read all articles. This question can also be considered for sentence (2). Is the arrival of each bishop from the the bishops a separate event (the distributive reading), or does a single arrival event encompass all the bishops (the collective reading). The quasi-logical form language extends the grammar of the logical form language to include rules that handle unscoped quantifiers, as in sentence (1), under-specified relations, as in sentence (2), as well as many other reference phenomena.

The logical form semantic representation is very well suited for modelling reference information where quantification is of key importance, and the discourse contains utterances where the exact scope of a quantifier is not clear. One of the biggest advantages to using logical form is that it allows us to use a large body of knowledge relating to first order logic. Although the Core Language Engine models some verb-structures ${ }^{4}$ using logical form and quasi-logical form, it does not attempt to perform any temporal ordering on the verb structures (as in the case of the timeline models from Section 2.4). Another slight deficiency in logical form is how it handles "fuzzy" quantifiers such as some, many, 
and few. In the Core Language Engine, the threshold defining the boundaries of these quantifiers is given a definite value. Common sense dictates that certain quantifiers, such as some, are fuzzy, and subject to contextual factors and personal preference.

\subsection{Conclusion}

This chapter has examined a number of semantic representations that are used in the fields of natural language processing and knowledge management, such as semantic networks, conceptual hierarchies, logical form and quasi-logical form. The benefits and potential drawbacks were outlined for each representation. Chapter 3 will examine models of human knowledge from the perspective of cognitive science. Chapter 4 will present a new model of semantic representation that attempts to address the shortcomings mentioned in this chapter by combining the representations into a modular multi-level system. This modular system is easier to maintain and allows multiple anaphora resolution algorithms to operate on a corpus simultaneously.

\footnotetext{
${ }^{4}$ Using first-order logic to model a verb phrase, intuitively, does not seem the most natural method to model that knowledge.
} 


\section{CHAPTER THREE}

\section{Working \& Long-Term Memory}

Although there is still great debate about the exact nature and structure of working memory and long-term memory, the majority of cognitive psychologists agree that the two forms of memory are distinct in their behaviour and capacity [Logie 1996]. The human mind does not have infinite time and infinite working memory capacity. If a natural language processing system is to more closely model how humans process language, it would make sense for that system to be constrained by the limitations and behaviour of human memory. It is the intent of this thesis to stimulate more interest in memory model approaches.

This chapter will outline some of the various views on working memory and longterm memory. It will briefly discuss the evolution of theories of short-term and longterm memory as well as current theories in human memory. Several modern psychological models of short-term and long-term memory will be elaborated on, as well as some memory models with a computational approach.

\subsection{Working Memory Models}

Some of the earliest work with respect to working memory can be found in the works of William James (1905) [Richardson 1996]. James described working memory, then termed primary memory, as being limited in capacity and volatile in nature. Primary memory was considered to be a distinct system from long-term memory (called secondary memory at the time). Information was retained in primary memory by rehearsal. Rehearsal was also used to move information to and from secondary memory. Within this model, primary memory did not control the flow or manipulation of the information, it only provided a medium of storage. 
Richardson continues by describing that during the 1960's, the theory of working memory, then termed short-term memory, was extended to include a control mechanism. This mechanism was responsible for the flow of information as well as processing. Instead of being used only for the storage of information, the space in short-term memory was shared with the processing of the control mechanism. Thus, in this model, there was a trade-off in working memory between processing power (in the control mechanism) and storage capacity.

The work of Baddeley on working memory is some of the most prominent. Gathercole and Baddeley outline in [Gathercole and Baddeley 1993] the structure and behaviour of Baddeley and Hitch's working memory model. Figure 3.1 gives a visual representation of their model. Gathercole and Baddeley state that the central executive is the most important component of the model. The central executive is responsible for controlling the flow of data within working memory, the retrieval of data from long-term memory and other memory systems, and the processing and storage of data. Baddeley expanded the model to include an episodic buffer in [Baddeley 2000]. In addition to the central executive, an additional two slave systems are also included in the working memory model, the phonological loop and the visuo-spatial sketch-pad. The phonological loop is responsible for verbal information while the visuo-spatial sketch-pad handles visuo-spatial information. Baddeley and Hitch used dual-task experiments in [Baddeley and Hitch 1974] to justify the separation of the two slave systems. They discovered that when a subject performed a verbal and a visual task concurrently, the individual could perform the tasks as efficiently as if the task were performed serially. When the number of tasks for a single slave system was increased to two tasks, the subject could not perform the tasks as efficiently as performing them one at a time.

\section{Limits of Working Memory}

The limits of working memory are as intensely debated as the structure of working memory. Early theories, such as Miller [Miller 1956], place specific limits on working memory. In 


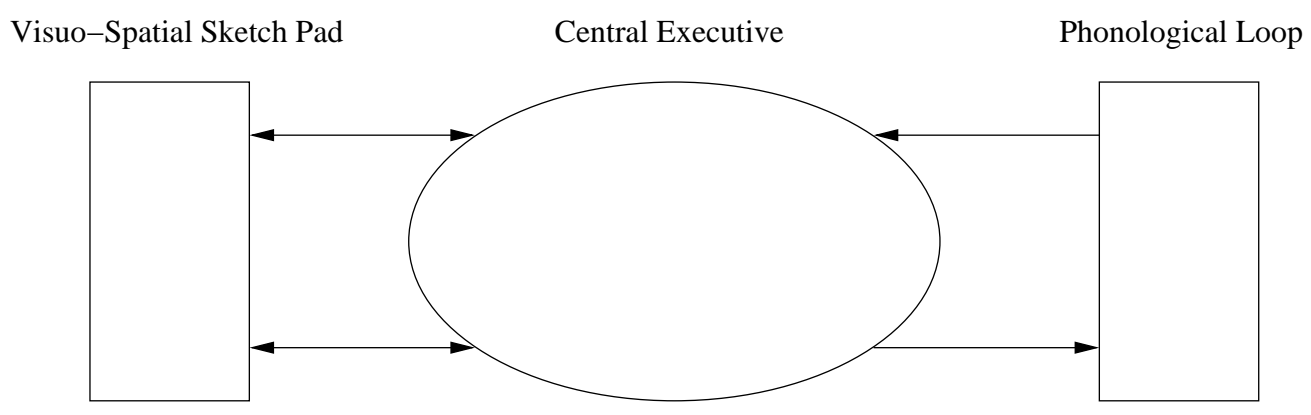

Figure 3.1: Baddeley and Hitch's Working Memory Model

Miller's view, working memory was seen as a short-term storage without any processing ability. More recent work, such as that found in [Baddeley 1986, Haarmann et al 2003], assumes that working memory is a multi-component system with processing capacity being inversely proportional to storage capacity. In [Baddeley 1990], Baddeley hypothesized that the span of working memory could partially be the result of the refresh-rate of items within the current memory span :

If we assume that memory fades, then the memory span will be determined by the number of items that can be refreshed before they fade away. That number, of course, will depend on how rapidly the trace fades and on how long it takes to articulate each item and hence refresh each memory trace.

\subsection{Long-Term Memory Models and Associativity}

Federmeier and Kutas mention in [Federmeier and Kutas 1999] that although long-term memory is an integral component of sentence processing, the exact nature of how workingmemory interacts with long-term memory information is still largely unknown. Just as there exists a debate as to the exact nature of working memory, differences in opinion also exist on how pieces of information are associated within long-term memory. Federmeier and Kutas outline two hypotheses, the independent association hypothesis and the associative symmetry hypothesis. The independent association hypothesis states that associations in memory are not bidirectional. That is, given that the recall of item $\mathbf{A}$ triggers 
the recall of $\mathbf{B}, \mathbf{A} \rightarrow \mathbf{B}$, does not necessarily imply that the converse, $\mathbf{B} \rightarrow \mathbf{A}$, is true. In the associative symmetry hypothesis, given $\mathbf{A} \rightarrow \mathbf{B}$ implies that $\mathbf{B} \rightarrow \mathbf{A}$. Using the independent association and associative symmetry hypotheses in the context of reading, when a word corresponding to a semantic concept is accessed through the reading of a sentence, the activation levels of neighbouring concepts may also increase.

\section{$3.3 \quad$ ACT-R}

The ACT-R theory of human cognition is rooted in ACT-E theory and ACT* theory which were introduced by John Anderson in [Anderson 1976, Anderson 1983], respectively. ACT-R models the interaction between two types of knowledge: procedural knowledge and declarative. Procedural knowledge involves rules that define human cognitive behaviour. Anderson formally calls these rules productions in [Anderson et al 2001]. Declarative knowledge encompasses factual information that defines behaviour of cognition, defined by Anderson as chunks. Examples of declarative knowledge are the sky is blue or snow is white. One of the major factors that influences cognitive performance in the ACT-R system is the granularity at which processing occurs. Production rules take at least $50 \mathrm{~ms}$ and at most $500 \mathrm{~ms}$ to fire.

\section{Procedural Long-Term Memory}

ACT-R is a goal-oriented system that uses productions to define the cognitive behaviour that acts upon declarative memory. Productions define actions such as retrieving information to be processed, as well as actions that define the manipulation of the retrieved information.

\section{Declarative Long-Term Memory}

In addition to the procedural memory, the ACT-R system also models declarative knowledge, that is, knowledge that is defined as being not factual and does not control the behaviour of cognition. Declarative memory is composed of chunks that are differentiated 
using a unique identifier. Each chuck has a type and may contain multiple slots, with each slot linking to additional chunks. A good example can be taken from [Group 2004]. Using the sentence the dog chased the cat, we can derive the following chunk of declarative memory:

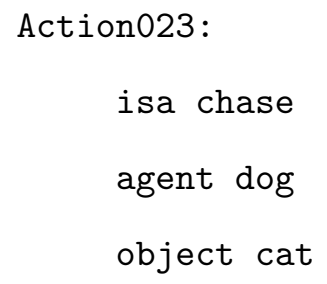

In this example, the type of the outer chunk is is a chase, and the two slots of the chunk are filled with the chunks agent dog and object cat.

\section{Declarative Memory Activation}

In the ACT-R system, the retrieval of declarative chunks in memory is governed by the speed at which they can be accessed. In [Anderson and Matessa 1997], Anderson defines activation equations that predict the power law of learning and the power law of forgetting. The activation level, $A_{i}$, of a declarative memory chunk ${ }^{1}$ is define as follows:

$$
A_{i}=B_{i}+\sum_{j} A_{j} S_{j i}
$$

where $B_{i}$ is the base level activation of the chunk $i, A_{j}$ is the activation of a chunk $j$ within the current focus of attention, and $S_{j i}$ is the strength of the association between chunk $j$ and chunk $i$. The base level activation, $B_{i}$, models the recency and frequency of activation of the chunk $i$, and thus has a factor of decay associated with it. $B_{i}$ is defined by ACT-R as:

\footnotetext{
${ }^{1}$ Anderson notes that the definition of chunks should not be confused with the definition from [Miller 1956]
} 


$$
B_{i}=\ln \left(\sum_{k=1}^{n} t_{k}^{-\omega}\right)
$$

where $t_{k}$ is the time since the $k \mathrm{th}^{2}$ use of the chunk $i$, and $\omega$ is the activation decay. In [Anderson and Matessa 1997, Anderson et al 1998], the value of $\omega$ is fixed at 0.5. In order for a chunk of declarative memory to be retieved and brought into the current focus, the threshold of activation, $\sigma$, must be met.

\subsection{Computational Models of Memory}

A survey of memory would not be complete without examining those models created from a computational perspective. In particular, this section will examine the models of memory presented by Schank in [Schank 1986] and Hunt in [Hunt 1973]. Although the types of memory described by Schank and Hunt may have much information overlap with previously described models, it is still relevant to examine computational models along with psychological approaches. To some respect, the idea of memory modelling has been largely ignored in the field of computational linguistics.

\section{Event Memory and Generalized Event Memory}

Event memory contains semantic knowledge for particular events experienced in a person's life. Schank states that events can be such things as going to Dr. Smith's dental office last Tuesday, and getting your tooth pulled or forgetting your dental appointment and having them call you up and charge you for it. As a specific event remains in memory longer, the exact details of the event begin to become less salient, and eventually, the event may become a more generalized event or it may disappear entirely.

Generalized event memory, as the name describes, is a more generalized version of the

\footnotetext{
${ }^{2}$ In [Anderson and Matessa 1997], the index $k$ is actually $j$. This was changed to prevent confusion with the index $j$ in the equation for $A_{i}$. In addition, $d$ is used as the decay rate, instead of $\omega$ and $A_{j}$ is used for the activation of node $j$ instead of $W_{j}$
} 
event memory described earlier. Generalized event memory is modelled as a portion of memory that contains abstract events, i.e. events that have occurred numerous times and thus have a template associated with them. As events from event memory are brought into short term memory the associated generalized event is also brought in to aid in cognitive processing. One of the results from this behaviour is that events from event memory will become less and less salient and the more generalized event will only remain.

\section{A Distributed Memory Model}

In [Hunt 1973], Hunt describes a model of memory that builds upon the basic memory model containing only short-term memory and long-term memory. As shown in Figure 3.2, Hunt adds an intermediate term memory structure that resides between short-term and long-term memory, and buffer memory which is analogous to sensory memory in other literature. Intermediate-term memory stores information about the current situation or episode, and thus intermediate-term memory is volatile like short-term memory. The buffer memory is the most volatile of the structures, storing stimulus from sensory input, such as auditory input, for only brief periods of time. In contrast to Baddeley's model of working memory where information flow within working-memory is controlled by the central executive, Hunt's model places control within the respective memory substructures, Hunt notes that in his distributed memory model each memory component is likely to be associated with varying anatomical areas of the human brain, but does not provide evidence.

\subsection{Neural Network Models of Associative Memory}

Neural Networks, or more correctly artificial neural networks, attempt to model the behaviour of neurons within the human brain. Some of the earliest work on the modelling of artificial neurons can be attributed to McCulloch and Pitts in [McCulloch and Pitts 1943] and Hebb in [Hebb 1949]. Since the early work of McCulloch and Pitts, the field of artificial neural networks has developed into a mature field with large amounts of research in 


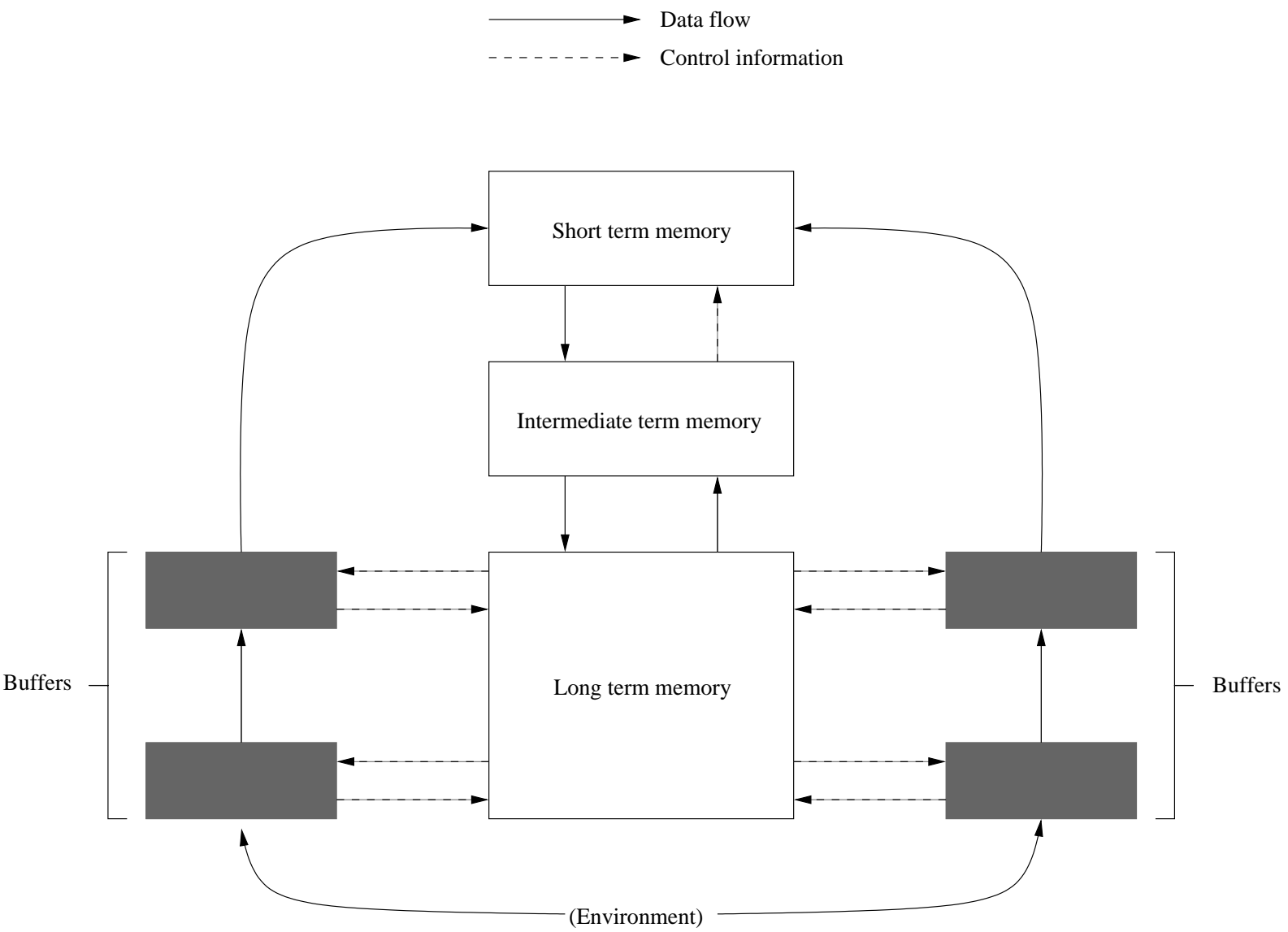

Figure 3.2: Hunt's Distributed Memory Model

areas such as network topology and neuron structure.

Artificial neurons are modelled after biological neurons. Axons send out signals to another neuron's dendrite. If the sum of the signals received by a neuron is greater than some threshold, then the neuron fires, sending signals along its axons to other neurons. Figure 3.3, adapted from [Russel and Norvig 1995], is an example of a typical biological neuron. Artificial neurons attempt to model neurons at the level of a single biological neuron. An artificial neuron does not attempt to model the actual physical chemical reactions occurring, rather, they model neurons at more of a cause and effect level.

A typical artificial neuron can be found in Figure 3.4. The input values for neuron $i$, $A_{j}$, can be thought of as the dendrites. The output value, $A_{i}$, is an axon. The strength of the link between node $i$ and some node $j \neq i$ is modelled using the $S_{j i}$ term. The bias 


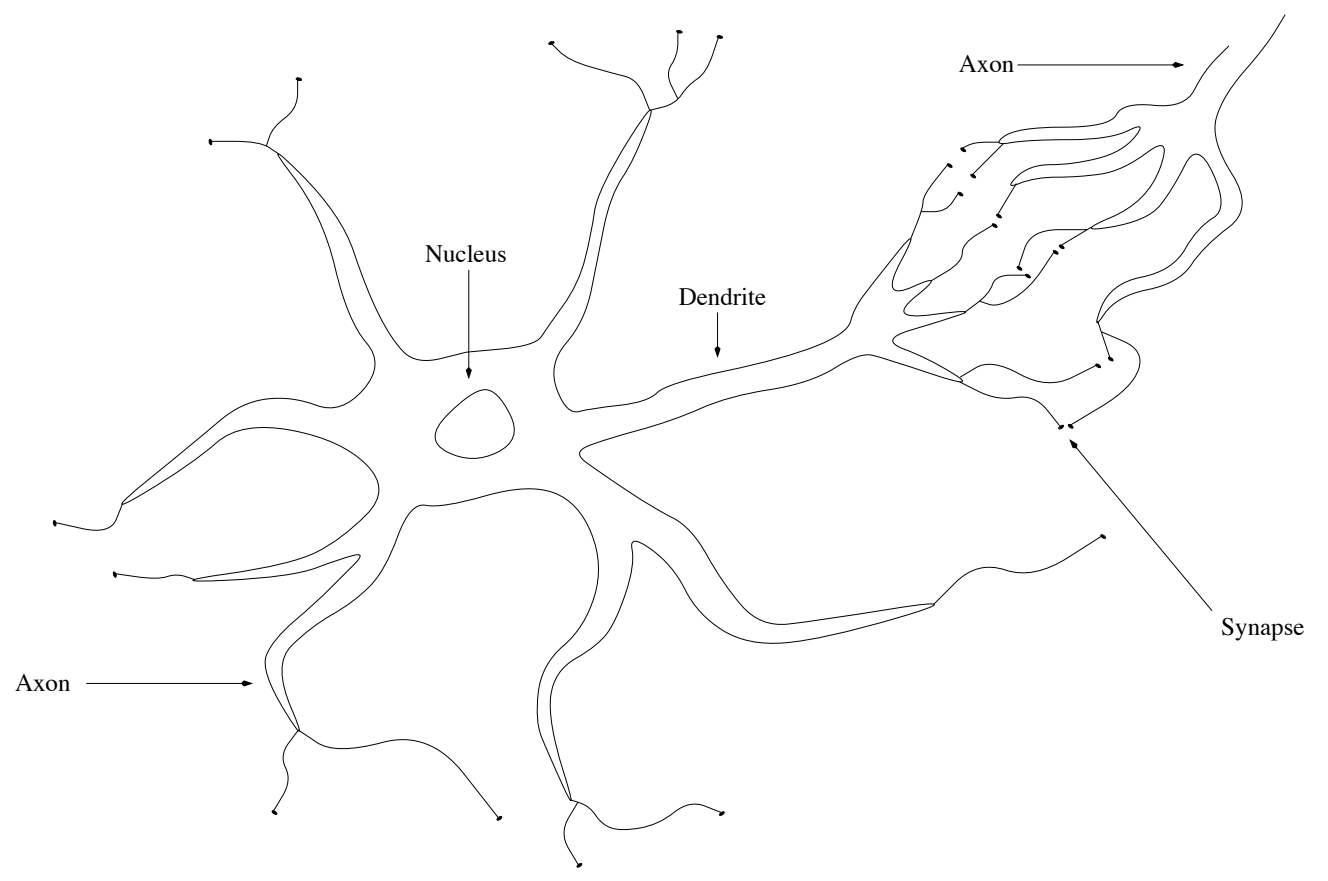

Figure 3.3: Biological Neuron

value, $B_{i}$, is often used to influence base-level activation, allowing $A_{i}$ to be non-zero. The activation function of a neuron takes into consideration the input values, $A_{j}$, and their associated link strengths, $S_{j i}$, and generates a result $f$. Typically, the neuron activation function $f$ for a node $i$ is defined as follows:

$$
f=B_{i}+\sum_{j=0}^{k} A_{j} S_{j i}
$$

The final portion of an artificial neuron is the threshold unit. If the total signal received by the neuron is greater than some threshold, the neuron will fire. The actual value of the threshold is defined by a threshold function, $g(f)$. The resulting output from the neuron, is represented as $A_{i}$. In general, $g$ can be any single-variable function, but the Equations 3.4-3.7 are some of the more commonly used. 


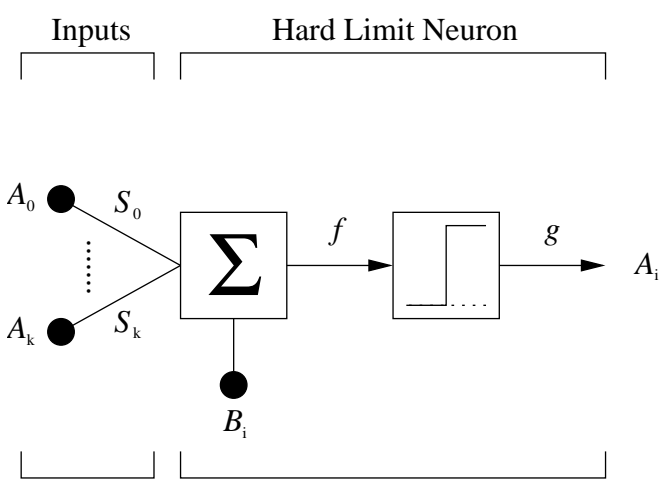

Figure 3.4: Multi-Input Neuron

$$
\begin{aligned}
\operatorname{hardlim}(n) & = \begin{cases}1 & \text { if } n \geq 0 \\
0 & \text { if } n<0\end{cases} \\
\operatorname{satlins}(n) & = \begin{cases}1 & \text { if } n>1 \\
-1 & \text { if } n<-1 \\
n & \text { if }-1<n<1\end{cases} \\
\operatorname{logsig}(n) & =\frac{1}{1+e^{-n}} \\
\operatorname{tansig}(n) & =\frac{e^{n}-e^{-n}}{e^{n}+e^{-n}}
\end{aligned}
$$

\section{Neural Network Topology}

A single set of inputs to a neuron is not very useful, or realistic. Of the $10^{11}$ neurons in the brain, each neuron is connected to $10^{4}$ other neurons. One of the most common configurations of neural network topology is shown in Figure $3.5^{3}$. This three-layer configuration allows arbitrary functions to be represented, and is the most commonly used in pattern matching applications.

\footnotetext{
${ }^{3}$ Each circle in the diagram represents a multi-input artificial neuron.
} 


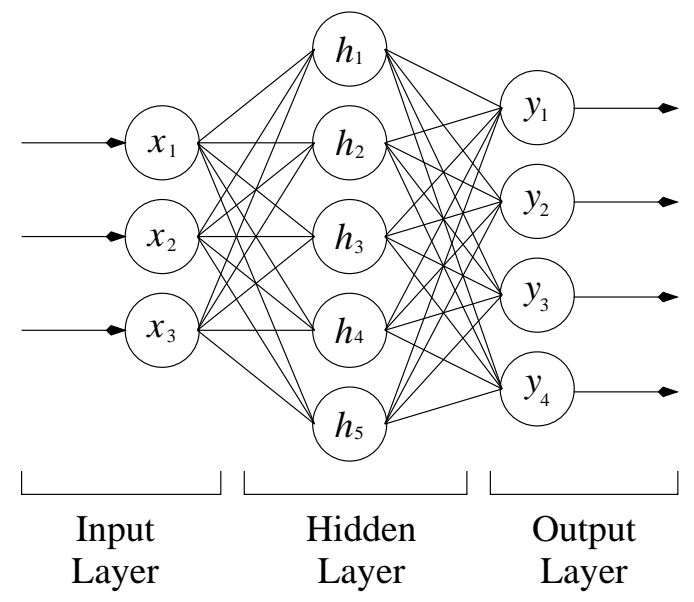

Figure 3.5: Multi-Layered Artificial Neural Network

\section{Hebbian Unsupervised Learning}

An artificial neuron by itself does not learn to perform complex tasks or match complex patterns. A learning strategy must also be applied. Two strategies of artificial neuron learning are supervised and unsupervised learning. This thesis will concentrate on the latter of the two learning strategies. Within the human brain, neurons are connected to many neighbouring neurons. The neuron must be capable of creating implicit associations without direct intervention. Before outlining how an artificial neuron can learn to make associations, it's important that neuron association be properly defined. Hebb's postulate from [Hebb 1949] states:

When an axon of cell $\mathrm{A}$ is near enough to excite a cell $\mathrm{B}$ and repeatedly or persistently takes part in firing it, some growth process or metabolic change takes place in one or both cells such that A's efficiency, as one of the cells firing $\mathrm{B}$, is increased.

Using Hebb's observation, the strength of the association between a neuron $i$ and a neuron $j$ can be realized using Equation 3.8 adapted from [Luger and Stubblefield 1998]:

$$
S_{i j}=S_{i j}+\alpha A_{i} A_{j}
$$


$S_{i j}$ is the strength of the association between neuron $i$ and neuron $j, \alpha$ is the learning rate of the semantic link, and $A_{i}$ and $A_{j}$ are the current activation levels of neuron $i$ and neuron $j$, respectively.

\subsection{Conclusion}

This chapter examined numerous theories on the structure and behaviour of human memory. The evolution of working memory and long-term memory was examined from the perspective of cognitive psychology. Modern theories on human memory were also examined. Models of human memory from computation, such as neural networks, event memory, generalized event memory, and the distributed memory model were discussed. Baddeley's work with respect to working memory has demonstrated that there is a trade-off of capacity versus processing power in working memory. The ACT-R model established how associations between concepts can be modelled as well as the activation of those concepts. The discussion on neural network demonstrated how human memory can be modelled by using the behaviour of biological neurons as its basis. In Chapter 6, the work of Baddeley, the ACT-R model of activation, and neural network theory will provide a basis for the behaviour and structure of a memory model. A second model of activation, called Thompson's model, will also be introduced in Chapter 6. The results of testing using the ACT-R and Thompson model will be discussed in Chapter 7. 


\section{CHAPTER FOUR}

\section{Theory on Combining Semantic Structures}

In Chapter 2, numerous semantic structures were outlined, as well as some of their shortcomings in their purest forms. A common thread was that each structure needed to be expanded in order to accommodate additional types of semantic information. The result is a structure that is possibly more complex, without the guarantee that the new structure can adapt to the dynamic nature of human knowledge and language. A structure that works today may not necessarily work tomorrow. From a software engineering perspective, modularizing the semantic structures makes the system easier to understand and easier to expand. As deficiencies are found in the semantic structures, new structures can be incorporated, and deficient structures can be removed.

\subsection{Linking Semantic Structures}

Intuitively, having completely disjoint semantic representations would not effectively model the nature of human knowledge. Research in human memory has shown that when concepts are activated in memory, related information may also be activated if the link between them is strong enough. Information from one semantic representation must somehow be linked to related information in another semantic representation. Semantic networks, which were introduced in Chapter 2, can be used to model the links between the semantic representations. Figure 4.1 expands on Figure 2.1 by connecting the semantic concept ship to various semantic representations such as quasi-logical form, conceptual graphs, conceptual hierarchy, and a timeline model. The information from a semantic representation could also be connected to other concepts nodes within the semantic network. In addition to the bare links, the strength of the links between concept nodes can also be considered by adding weights to each link. In theory, the more often the concept node in the semantic network is activated, the stronger the link will be to other concept nodes. 
This is similar to the behaviour of synapses originally theorized by Hebb in [Hebb 1949]. In addition, activation levels for the concept nodes can be modelled using activation level models from artificial neural networks and ACT-R theory. The topology of the resulting network differs from the traditional artificial neural network topology in that there is no distinct input, hidden, or output layers. Rather, the concept nodes are connected in a non-specific fashion. The resulting network is commonly called a localist network in other literature. Single concepts are represented as a single node within the network. This representation differs from networks like artificial neural networks, where a single concept is represented as activations across a set of nodes, and nodes can represent more than one concept.

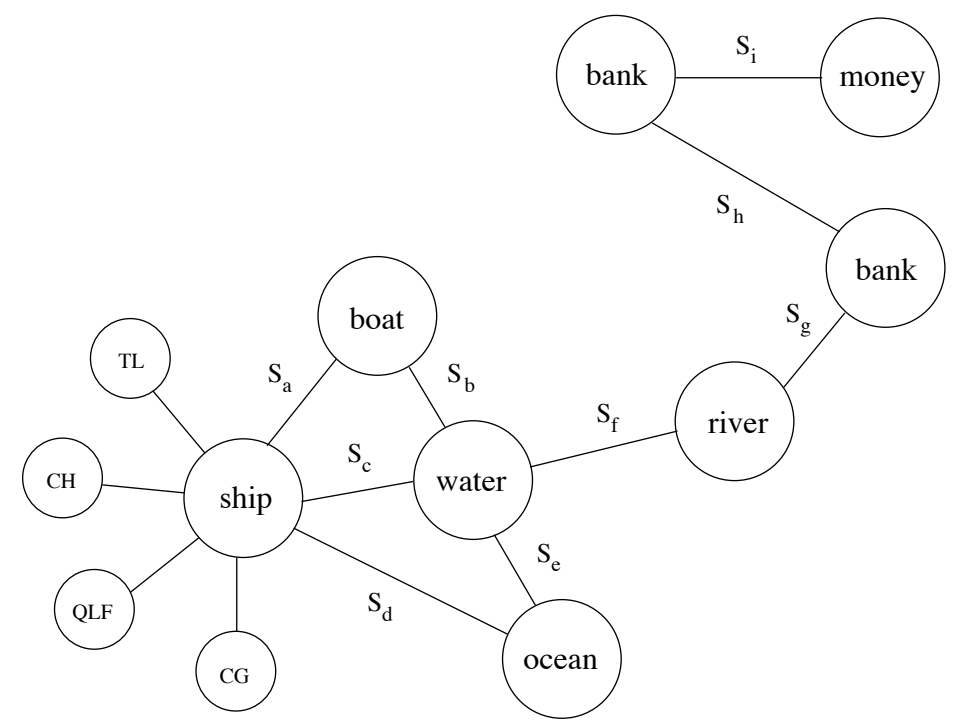

Figure 4.1: Semantic Network Linking Semantic Representations

\section{Implicit Semantic Links}

It is plausible that knowledge which is represented using one semantic representation is often intertwined with the knowledge within another. Figure 4.2 shows a good example of how multiple semantic representations collectively model the sentence Once there was a shoemaker who worked hard and was very honest. Notice that certain information such 
as shoemaker:1 and work:1 occur in more than one structure. We would like to some how connect this information together, and a semantic network would be a good method to achieve these connections. Concepts in the semantic network would be automatically linked due to the information within their semantic representations.

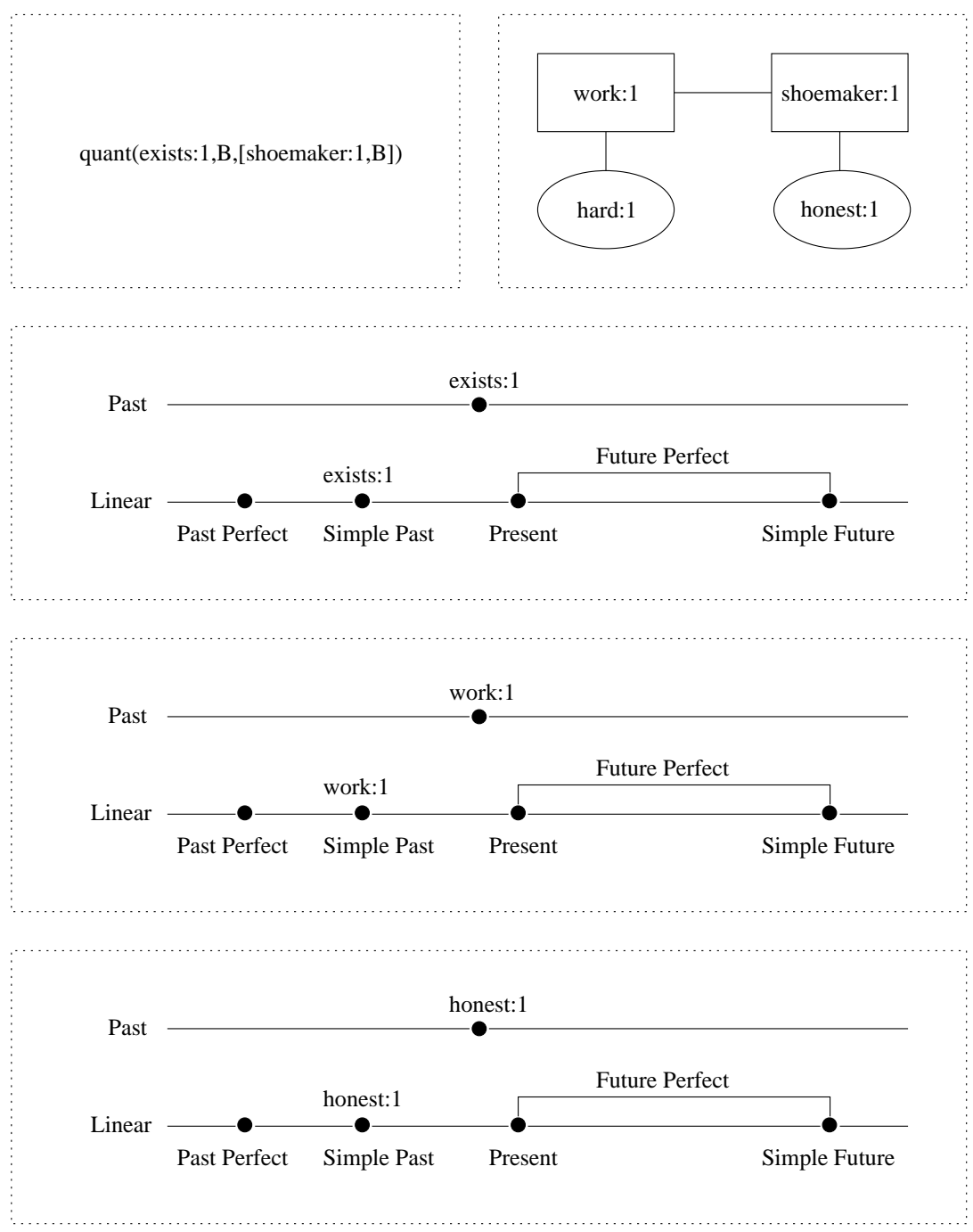

Figure 4.2: Multiple Semantic Representations 


\subsection{Modularization and The Human Mind}

Creating a modularized model of semantic structures is not only a good engineering technique, it is also supported as model of the behaviour of the human mind by such psychological literature as [Foder 1983]. Modularity of mind theorizes that parts of the human mind are modular in nature and act autonomously with respect to other modules in the mind. To some degree, the modularity is thought to be genetically determined. Foder's model of human mind requires that modules be specialized in their domain, encapsulate their information from other modules, and have limited outputs to other modules. These requirements are a natural result of the model described in this chapter.

\subsection{Conclusion}

This chapter has introduced a modular model of semantic representation that addresses the drawbacks of existing semantic representations by connecting them using a semantic network. This model allows new semantic representations to be added and removed from a system without impacting the existing ones. It also addresses the desire to expand a semantic representation to deal with new forms of knowledge. Instead of expanding to a current semantic representation, a new semantic representation can be created and plugged into an existing system. This modular separation of semantic representations also permits the development of anaphora resolution algorithms for each semantic representation independently. It is apparent that in order to combine the semantic structures from Chapter 2 using a semantic network, a theory of the exact nature of the network must be considered. The link strength between concepts needs to be modelled, as well as the activation levels for each node. Chapter 3 discussed theories on how link strengths and activation levels are determined in the ACT-R system [Anderson et al 2001] and using traditional artificial neural network theory. Chapter 6 will outline how activation theories will be used in conjunction with a semantic network to define the behaviour of the model proposed in this chapter. 


\section{CHAPTER FIVE}

\section{Anaphoric Reference and Reference Resolution}

Anaphoric reference is a linguistic mechanism with which reference can be made to objects that have been introduced at an earlier point. References are typically made with pronouns or different variations of definite/indefinite articles within a noun phrase. Anaphoric references are also used to reference verb phrase structures. Anaphora, especially in the case of pronouns, often can be resolved by scanning backwards through a corpus of text until the first noun phrase that matches such features as number and gender is found, although Barbara Grosz demonstrated in [Grosz 1977] that this technique can break down.

\subsection{Types of Anaphoric Reference}

Traditionally, anaphoric reference is observed in the use of pronouns such as he, she, or it. But within recent decades, there have been numerous proposals to extend the definition of anaphoric reference to include other linguistic phenomena such as verb phrase ellipsis [Grosz 1977, Hardt 1997, Nash-Webber and Reiter 1977, Ginzburg and Cooper 2001], presupposition [Piwek and Krahmer 2000, Geurts 1999], and temporal anaphora [Partee 1984]. In the context of this thesis, the domain of anaphoric references will be restricted to pronominal references of noun phrases.

\subsection{Problems in Anaphora Resolution}

As stated before, in many cases resolving the antecedent for a pronoun is as simple as searching backwards in a body for the first noun phrase that matches based on such attributes as gender or number. But in some cases, a more complex model discourse must be modelled in order to resolve a pronoun reference: 
John had a son named Bob. His son is an excellent skier.

In this example, a knowledge base about parent-child relationships must be known in order to resolve the reference implied the possessive pronoun his. Even trivial references can be more complex by introducing existential quantifiers, as illustrated by Partee in [Partee 1984]:

Every farmer who owns a donkey beats it.

The pronoun it does not just reference a single donkey, the pronoun references multiple instances of a donkey. The next example, adapted from [Sidner 1983], demonstrates where this method of resolution can break down:

My neighbours 1 have a monster Harley $1200{ }_{2}$. They 3 are really huge but gas efficient bikes.

In the second sentence, if an individual was to read just the pronoun they, their initial preference for the reference may not be a monster Harley 1200 based on number alone. In this context, a common preference for the pronoun they would be my neighbours. After reading the remainder of the second sentence, it is apparent that this conclusion was incorrect. Given the additional context, common knowledge concludes that the neighbours are not motorcycles

\subsection{Anaphora Resolution Algorithms}

Determining the antecedent of an anaphor is central to the study of anaphoric reference. Over the past few decades much research has involved creating computational methods to resolve these references. Works such as [Sidner 1979, Sidner 1983], [Grosz 1977, Grosz and Sidner 1986], and [Carter 1985, Carter 1990] have concentrated on the study of discourse and the theory of anaphora within a discourse, while [Hobbs 1986, Brown 2003, Mitkov 1998] have focused more specifically on pronominal anaphora resolution. The next 
few sections will outline Carter, Hobbs, Brown, and Mitkov's approaches to pronominal anaphora resolution.

\section{Hobbs' Approach and Brown's Algorithm}

In [Hobbs 1986], Hobbs outlines a simple algorithm for the resolution of pronouns, and although naïve, it provides goods results. The algorithm works by starting at the location of the pronoun and working back through the parse tree in a breadth-first manner until a suitable antecedent match based on gender and plurality is found. When tested on 300 occurrences of references in selected corpora, the algorithm had a success rate of $88.7 \%$ in resolving the anaphoric reference. Hobbs notes though that in over half of the cases, there was only one plausible antecedent.

Hobbs analyzed the results further and went on to consider the results for the cases when there was more than one plausible antecedent. Of the 132 cases where an antecedent conflict existed, 98 were resolved by the algorithm, thus a $74.4 \%$ success rate. Hobbs goes on to improve the naïve algorithm by adding simple restrictions for resolving pronouns, such as dates can't move, places can't move, and large fixed objects can't move. Without these restrictions, the success of the resolution algorithm was, $81.8 \%$, overall. When the selectional restrictions were used, a $91.7 \%$ success rate was achieved.

In [Brown 2003], Brown outlines an algorithm for resolving noun phrase references that is a variation on Hobbs algorithm. Figure 5.1 illustrates the algorithm in pseudo-code ${ }^{1}$.

Brown's algorithm has the benefit of not specifying how a reference is resolved when there are multiple antecedents for a single noun phrase, which consequently, allows the implementor to choose how the antecedent can be resolved.

\section{Carter's Approach}

In his PhD thesis, [Carter 1985], Carter describes in [Carter 1990] the approach used in the SPAR system . The SPAR system initially starts by resolving semantic and syntactic

\footnotetext{
${ }^{1}$ In Figure 5.1, NP is an abbreviation for noun phrase.
} 
IF the NP is a proper name THEN

ATTEMPT to identify the reference in the knowledge base

IF no antecedent is found THEN

CREATE a new reference in the knowledge base

ELSE IF the NP is an indefinite NP THEN

CREATE a new reference in the knowledge base

ELSE IF the NP is a reflexive pronoun then

SET the reference to the subject of the clause

ELSE IF the NP is a pronoun THEN

CHECK NPs that precede for number/gender/person agreement

check NPs in previous sentences in the same manner

ELSE IF the NP is a definite NP THEN

CHECK NPs that precede for number/gender/person agreement

CHECK NPs in previous sentence in the same manner

IF no is antecedent is found THEN

CREATE a new reference in the knowledge base

Figure 5.1: Brown's Anaphora Resolution Algorithm

issues without concerning itself with potential anaphoric references. Multiple structures can result from this process depending upon the word-sense of the words within a sentence. Given the sentence He picked up a jack, Carter theorizes two possible structures. One structure where jack is interpreted as a playing card, and the second where it is a tool used to raise an automobile. According to Carter's algorithm, the pronoun he is left unbound, and will be dealt with in further stages.

After the initial structures are generated, they are reprocessed and given scores based on factors such as repeated relevant information and its influence on syntactic structure. For example, in Figure 5.2, (b) would be given a higher score than (a) because a telescope is used for seeing things, thus it is more highly related to the verb saw than the noun phrase a man. After assigning scores, the algorithm proceeds to use anaphora resolution rules which Carter describes as being similar to those found in [Sidner 1979]. 


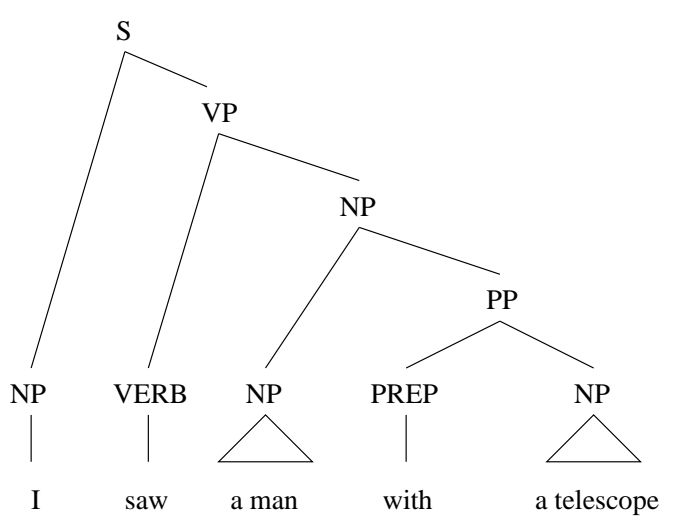

(a)

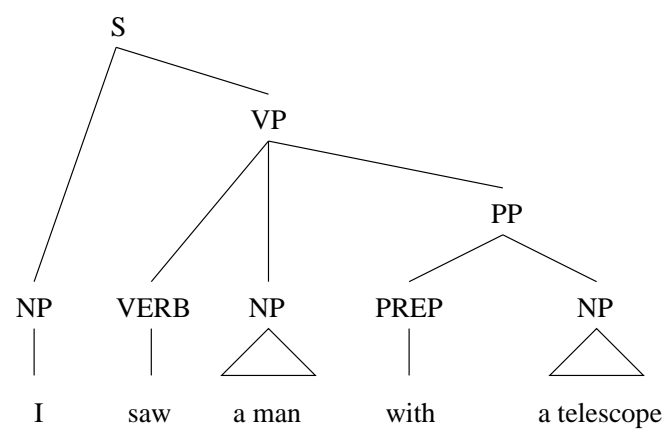

(b)

Figure 5.2: Two Parsings of I saw a man with a telescope

\section{Mitkov's Work}

In [Mitkov 1998], Mitkov outlines an anaphora resolution algorithm that uses scoring factors to determine a plausible antecedent to a reference. The scoring factors are based on the analysis of what Mitkov terms indicators. Indicators can be such things as the definiteness of the possible antecedent, the givenness, indicating verbs, lexical reiteration, prepositional position, and referential distance. The domain of possible scores is $\{-1,0,1,2\}$, with varying values being chosen for each indicator class. Figure 5.3 outlines Mitkov's algorithm in pseudo-code format. Mitkov claims a success rate of $89.7 \%$ with this algorithm.

\subsection{Resolution Failure}

Many resolution algorithms make the assumption that the antecedent of an anaphora must be resolved. Levine hypothesizes in [Levine et al 2000] that there are conditions under which readers fail to resolve the anaphoric reference, yet are still able to comprehend the text. Levine showed that if the antecedent was salient and distant enough from the point of reference, readers were content with not resolving the reference if it was not disruptive to the comprehension of the text. Although this finding could have a big impact on how 
EXAMINE the current sentence and the two preceding sentences

SELECT the noun phrases which agree in gender and number

APPLY the antecedent indicators to each candidate and assign scores

IF two candidates have an equal score THEN

SELECT candidate with the higher score for immediate reference

IF immediate reference does not hold THEN

SELECT the candidate with higher score for a collocational pattern

IF collocational pattern suggests a tie or does not hold THEN

SELECT the candidate with higher score for indicating verbs

IF this indicator does not hold THEN

SELECT the most recent candidate

Figure 5.3: Mitkov's Anaphora Resolution Algorithm

anaphora resolution algorithms will work in the future, it does not begin to explain what a comprehensible piece of text is.

\subsection{Conclusion}

This chapter has given an overview of the problem of anaphoric reference with respect to pronouns, as well as algorithms that address reference resolutions. Chapter 6 will describe an anaphora resolution algorithm that uses the theories on working memory and activation, introduced in Chapter 3, to create a list of possible antecedents for a pronoun. The algorithm will then use the idea of feature set scoring from Carter to form a basis for resolving conflicts when multiple plausible antecedents exist. Chapter 7 will outline the results of the algorithm when tested on The Three Brothers and the Rumpelstiltskin corpora using the ACT-R model and the Thompson model of activation. 


\section{CHAPTER SIX}

\section{System Modelling}

If you don't gosub a program loop, you'll never get a subroutine.

- KRYten (Justice)

Throughout Chapters 2, 3, and 4, varying theories on semantics representation, the structure of human memory, and its behaviour have been discussed. This chapter will focus on combining parts of these various theories to solve the problems of anaphora resolution outline in Chapter 5. The model of long-term memory and working memory will be outlined as well as the grammar rules and their interaction with the memory models. The algorithm for anaphora resolution will also be discussed. For the purposes of this thesis, the semantic network will be the only semantic representation that is implemented. The implementation of other semantic representations, such quasi-logical form and concept graphs, will be deferred to future work. The implementation and subsequent testing of the semantic network will provide baseline results from which this future work can be compared to.

\subsection{Modelling Long-Term Memory}

In this thesis, long-term memory will be modelled using a combination of the semantic network theory described in Chapter 2 and the neural network theory described in Chapter 3.

\section{Semantic Networks for Long-Term Memory}

Semantic networks are undirected graphs with strengths associated with the links between nodes. Within the context of this thesis, as was described in Chapter 4, semantic networks 
will not be used directly to store semantic information, rather, they are used to link the other existing semantic structures (i.e. conceptual graphs, classification hierarchies, event timelines, and quasi-logical form). This decision is intended to achieve a behaviour similar to that observed in neural network theory. As the activation levels of a node within the network increase, the activation of neighbouring nodes will also increase. Semantic markers will be used to uniquely identify semantic structures and concepts.

Table 6.1 outlines the Prolog predicates. In the nn_semNode predicate, SemMarker signifies the semantic marker for the node, Activation holds the current activation, and ActHistory contains the activation history of the node. In the nn_semLink predicate, SemMarker:1 and SemMarker:2 identify the two structures or concepts being linked, and Strength is, of course, the strength of the link. The nn_semNode and nn_semLink predicates provide all that is required to build and modify a semantic network.

\begin{tabular}{|c|c|}
\hline Predicate & Description \\
\hline \hline nn_semNode(+SemMarker,+Bias,+Activation,+ActHistory) & Semantic Node \\
\hline nn_semLink(+SemMarker $: 1,+$ SemMarker $: 2,+$ Strength) & Semantic Link \\
\hline
\end{tabular}

Table 6.1: Semantic Network Predicates

\section{Activation Level Models}

The two different models of semantic node activation levels will be used and tested in this thesis: (1) The ACT-R model for activations of declarative memory, and (2) a model derived empirically, called Thompson's model. From the ACT-R model, Anderson's model for the activation level and activation decay for declarative memory will be used, and will be based on Equations 3.1 and 3.2, as described in Chapter 3. Since the activation level of the ACT-R equation is unbound, the sattins threshold equation, Equation 3.5, will be applied to bound the resulting activations to the range $-1 \geq A_{i} \leq 1$.

Thompson's model will be based on Equation 3.3 for the activation function of a semantic node, Equation 3.7 for threshold function, and the following equation for the fading the activation: 


$$
A_{i}=\omega A_{i}
$$

where $\omega$ is the decay rate of node $i$ 's activation. The base level activation of node $i$ will be set to $B_{i}=0.0$. It must be noted that the decay equation for Thompson's model is not applied during node activation, as in the case of the ACT-R model. Rather, decay will occur, due to mental processing in working, which will be described in more detail later in this chapter.

\subsection{Working Memory}

If the activation level of node $i$ is greater than some activation threshold, $\sigma$, node $i$ will be brought from long-term memory into working memory. Working memory acts as a repository for concepts that are easily accessible for mental processing.

\section{Working Memory Structure}

In this thesis, working memory does not contain the actual structures that represent the currently active concepts, rather, working memory is conceptualized as a list containing semantic markers. The semantic markers act as links to the concepts within long-term memory. As semantic concepts are activated, they are placed within the list representing working memory, and as they decay, they are removed from the list.

\section{Semantic Node Behaviour}

The working memory model that was modelled is based on the model described by Baddeley in [Baddeley 1986, Baddeley 1990, Gathercole and Baddeley 1993]. From Baddeley, the theory on working memory capacity was used. The limits of working memory will be based on the contention between storage capacity and processing time. Storage and processing are inversely proportional to each other, and thus, processing will affect how 
quickly active concepts in working memory will fade. In this thesis, processing will be restricted to the application of grammar rules only. As grammar rules are used (i.e. mental processing), the activation levels of concepts in working memory will fade. This fading effect of grammar rules gives the storage versus processing behaviour described in Baddeley's model. When the activation level of node $i$ falls below $\sigma$, node $i$ will be removed from working memory.

\section{Semantic Link Behaviour}

As will also be described in Chapter 7, the semantic links between semantic nodes do not exist when the system is initialized. Semantic links are created between the nodes in working memory after each sentence is parsed and are given an initial link weight, $S_{i j}$ and $S_{j i}$ (a semantic link for each direction). If the links already exists between nodes, new links will not be created.

Although the strengths of links between the nodes are created with the same initial value, they are updated independently after this creation. When a semantic node $i$ is activated into working memory, the new strength of the link to node $j$ is updated. The equation for Hebbian learning, Equation 3.8, will be used for calculating the new semantic link strength:

$$
S_{i j}=S_{i j}+\alpha A_{i} A_{j}
$$

\subsection{Prolog Model of Working Memory}

The Prolog model of working memory will be a functor, wm_workingMemory/1, with a single list as an argument. The list will contain the semantic markers of the concepts that are currently active in working memory. The current activation level of a concept will not be contained within the list, rather, the semantic marker will be used to look-up the activation in the semantic network described earlier. For example, given that concepts rimmer:1, lister:1, and kryton:1 are active in working memory, the following functor would 
result:

wm_workingMemory $([$ rimmer : 1 , lister : 1 , kryton : 1] $)$

\subsection{English Grammar Rules}

One of the major advantages of using Prolog as an implementation language, is that it allows the use of a Definite Clause Grammar to specify grammar rules. Definite Clause Grammars also reduces the amount time required to code grammar rules by eliminating the need to specify mechanism for consuming words from a sentence while parsing. For example, rather that using the rule

$$
\operatorname{adj}([\text { long } \mid B], B) \text {. }
$$

to process the adjective long, we can use the Definite Clause Grammar rule

$$
\text { adj } \rightarrow \text { [long] }
$$

Definite Clause Grammars are much more elegant because, notationally, they are very similar to context-free grammars. The result being that the source code will be more readable, easier to maintain, and less prone to errors.

The cost associated with using a Prolog grammar rule will be explicitly modelled within the grammar rules. Prolog allows us to add goals to Definite Clause Grammar rules that, when expanded into regular Prolog predicates and clauses, do not consume words from the input string while parsing. As an example, consider the following grammar rule:

$$
\operatorname{sent}(\operatorname{sent}(\mathrm{NP}, \mathrm{VP})) \rightarrow \mathrm{np}(\mathrm{NP}), \mathrm{vp}(\mathrm{VP})
$$

Adding a processing cost, the resulting rule would be something similar to the following:

$$
\operatorname{sent}(\operatorname{sent}(N P, V P)) \rightarrow\left\{n_{-} \_ \text {fadeNodes }\right\}, n p(N P), v p(V P)
$$


Here, the predicate $\mathrm{nn}_{-}$fadeNodes is a predicate that updates the activation levels of concepts in working memory.

The placement of the cost predicate within the grammar rule is important. By placing $\mathrm{nn} \_$fadeNodes at the front of the rule, the cost is incurred as soon as the rule is used. This placement creates the behaviour that as more backtracking is performed on grammar rules, the more complex the processing. Concepts will fade much more quickly from working memory when backtracking occurs, as opposed to no backtracking. If nn_fadeNodes was placed at the end of the grammar rule, the cost would only be incurred after the successful completion of a grammar rule.

\subsection{Annotated Parse Tree Model}

In this thesis, the modelling of parse trees will be an extension to the model found in [Sterling and Shapiro 1999], where parse trees are stored in an embedded-functor form. For example, given the following Definite Clause Grammar rule

$$
\text { sent } \rightarrow \text { np, vp. }
$$

the equivalent Definite Clause Grammar rule with parse trees embedded would be

$$
\operatorname{sent}(\operatorname{sent}(\mathrm{NP}, \mathrm{VP})) \rightarrow \mathrm{np}(\mathrm{NP}), \mathrm{vp}(\mathrm{VP})
$$

Sterling and Shapiro's parse tree model will be extended to also include the antecedent for pronoun references. Parse trees of the form

$$
\operatorname{noun}(\mathrm{N})
$$

would take the following form for pronouns, where "Ant" is bound a plausible antecedent for the pronoun or "null" if no antecedent exists.

$$
\operatorname{noun}(\mathrm{N}, \text { ant }(\text { Ant }))
$$

Since this extension to the parse tree model still adheres to the syntax of the original model, existing pretty printers can be used on the model without any modifications. Figure 6.1 shows two example parse trees with an unresolved and a resolved antecedent. 


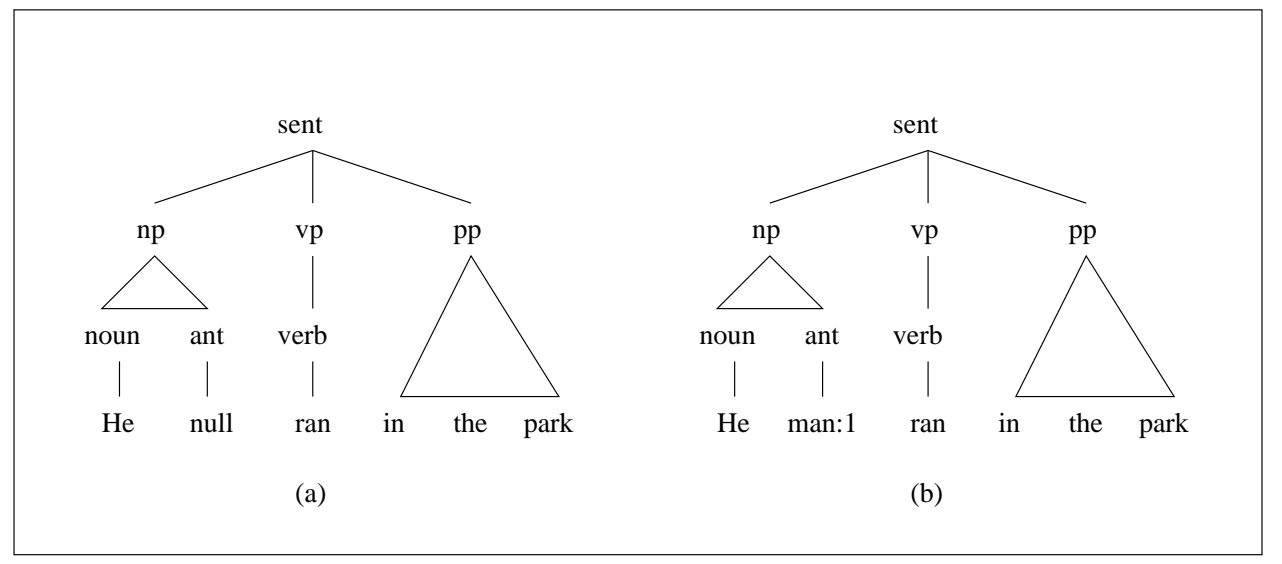

Figure 6.1: Annotated Parse Trees with (a) Unresolved and (b) Resolved Antecedents

\subsection{Lexical Feature Sets}

Lexical feature sets have been used in various theories of natural language processing such as Generalize Phrase Structure Grammars [Bennet 1995], in the Core Language Engine [Alshawi et al 1989], and the LangEng project [Brown et al 2001]. Features sets allow parsers to restrict parsing based on set of semantic attributes inherent to certain words. For example, in addition to semantic meaning, nouns also have attributes that imply the gender, person perspective, and plurality of the noun. The feature set syntax used in this thesis will be an extension on feature set syntax of the LangEng Project.

Each noun entry will contain two feature sets. The first feature set, the lexical feature set, will contain lexical entries such as the noun's case, category, and so forth, The second feature, called the semantic feature set, will contain semantic features such as the gender, number, and person perspective. The following is an example of a noun entry with the above features sets:

noun $([$ case : nom, cat : np], [gender : masc, num : sing, person : 3] $\rightarrow[$ he]

Feature sets are used by anaphora resolution algorithms to resolve references when multiple antecedents exist. Section 6.7 will discuss how the feature sets are used with an anaphora resolution algorithm. Tables 6.2 and 6.3 illustrate the noun feature sets that 
were used in this thesis.

\begin{tabular}{|c|c|c|}
\hline Feature & Value Set & Description \\
\hline \hline sem & prolog atom & semantic marker \\
\hline cat & $\{n p\}$ & marks lexical category \\
\hline case & $\{$ nom, acc, gen, dat, abl, loc, tmp $\}$ & morphological case \\
\hline sound & $\{$ soft, hard $\}$ & first consonant sound \\
\hline type & $\left\{\right.$ common, proper, gerund,pronoun, $r l f x \_$pronoun $\}$ & noun type \\
\hline
\end{tabular}

Table 6.2: Lexical Feature Set

\begin{tabular}{|c|c|c|}
\hline Feature & Value Set & Description \\
\hline \hline gender & $\{$ masc, femn, neut $\}$ & gender of a noun \\
\hline num & $\{$ sing, plur, mass $\}$ & plurality of a noun \\
\hline person & $\{1,2,3\}$ & person perspective \\
\hline
\end{tabular}

Table 6.3: Semantic Feature Set

\subsection{Anaphora Resolution Algorithm}

In Chapter 5, various anaphora resolution algorithms were discussed. A common thread between all the algorithms is that in the absence of multiple antecedents for a reference, the correct antecedent is identified, with the exception of those cases outlined in [Levine et al 2000].

The algorithm that was implemented is combination of the ideas outlined by Brown in [Brown 2003], Carter in [Carter 1985, Carter 1990], and the results from Koh and Clifton in [Koh and Clifton 2002]. Brown's algorithm will provide resolution for references that only have one antecedent. From Carter, the idea of scoring factors to influence how conflicts between lexically identical words are resolved was used.

The scoring factor for a semantic concept $i$ will be a combination of the current activation of node $i$ within working memory and relative similarity of the semantic feature set of concept $i$ to the semantic feature set of a pronoun $j$ : 


$$
\text { Score }_{i}=A_{i}+F_{i j}
$$

where $F_{i j}$ a score based upon the relative similarity of the feature sets of concept $i$ and pronoun $j$. The value of $F_{i j}$ is computed by starting with an initial score of 1.0. Each entry in the semantic feature set of concept $i$ that matches an entry in pronoun $j$ increases $F_{i j}$ by a factor of $\phi$, and each entry that does not match decreases $F_{i j}$ by a factor of $\psi=\frac{1}{\phi}$.

The current activation of node $i$ will influence whether it is compared to pronoun $j$ in the anaphora resolution algorithm. The algorithm will ignore concepts with an activation of less than $\sigma$. Figure 6.2

FIND the semantic concepts currently in working memory IF (at least one concept was found) THEN

FOR ( each concept $i$ found) DO

SET Score $_{i}=1.0$

FOR (each feature in the feature set of concept $i$ ) DO

COMPARE the feature value to the feature of the pronoun IF (the values match) THEN

SET $_{\text {Score }}=$ Score $_{i} * \phi$

ELSE

SET Score $_{i}=$ Score $_{i} * \psi$

ADD the activation level $\left(A_{i}\right)$ of node $i$ to Score $_{i}$ FIND the concept with the highest value for Score $_{i}$ SET the antecedent of the pronoun to that concept ELSE

SET the antecedent of the pronoun to NULL

Figure 6.2: Pronoun Reference Resolution Algorithm Pseudo-code

\subsection{Summary of Chapter}

This chapter has examined models for long-term and working memory and how they were realized in Prolog. It has also given a general overview of the format of the grammar rules that are used as well as the feature sets and annotated parse trees that accompany the 
rules.

In Chapter 7, the model described in this chapter will be tested against a number of corpora with varying decay rates for the semantic nodes. 


\section{CHAPTER SEVEN}

\section{System Testing}

This chapter will cover the methodology and procedures used for testing the anaphora resolution algorithm described in Chapter 6 using the activation model from ACT-R and the Thompson activation model. Various activation decay rates will be tested for each model. The results will be compared to human-based resolution.

\subsection{Overview of Testing Methodology}

The anaphora resolution algorithm will be tested against a corpus selected from the Grim Brothers library found at [Ockerbloom 2006]. The selected body of text was slighted modified from their original form to facilitate ease of parsing while retaining the spirit of reference placement. The modified text can be found in Appendix 8.3.

Each corpus will be tested independently and not have influence on the tests of the other two corpora. That is to say, the working memory, and long-term memory will be reset to a default configuration for each test phase. The bodies of text will be tested a number of times each with different decay rates, $\omega$, for the semantic nodes within long-term memory.

\subsection{Testing Platform}

The testing was performed on a $1.8 \mathrm{GHz}$ PowerPC G5 1.25 GB RAM under Mac OS X v10.4.4 using SWI-Prolog v5.4.7.

\subsection{Decay Rates}

Since the decay rate, $\omega$, affects the activation levels in the ACT-R model differently than those in the Thompson model, different sets of decay rates were chosen. The sets of decay 
rates were chosen in such a way that they presented a broad spectrum of the behaviour for each model. The value of $\omega$ ranged from values that caused short activation times, i.e. fast activation decay, to values that caused low activation, and thus caused the contents of working memory to be quite high.

In the ACT-R model, the values of $\omega$ ranged from 0.05 to 0.30 . The decay rate of $\omega=0.5$, which is used in ACT-R, was not chosen because preliminary testing showed that the value caused an extremely high level of decay, which resulted in a large number of pronouns being unresolved. This extreme decay is most likely due to the fact that the ACT-R model may not be $100 \%$ compatible with a neural network-type model.

The decay rates in the Thompson model ranged from $9.90999 \times 10^{-1}$ to 1.0 . Although, a decay rate of $\omega=1.0$ would imply no decay, that is not actually the case. Since the current activation is also based on neighbouring semantic nodes and the weight between the nodes, a certain amount of decay will still occur.

\subsection{Default Memory Configuration}

Initially, the contents of working memory were empty. This initial state of working memory was represented in Prolog by an empty list as the argument of the wm_workingMemory functor:

wm_workingMemory([])

Long-term memory, was represented as a neural network, initially contained nodes for all possible nouns that can be parsed by the system. Each node, $i$, had an initial activation $A_{i}=0.0$, and bias $B_{i}=0.0$. The links between nodes did not exist, rather, they were created as describe in Chapter 6.

\subsection{Testing Procedure}

The ACT-R and Thompson activation models of system were tested against all of the sentences from the The Three Brothers corpus in sequence using various rates of decay. 
The following items were tabulated during testing:

- results of the anaphora resolution algorithm were tabulated against the expected results outlined in the tagged corpora of Appendix B

- the maximum capacity of working memory across all decay rates for each activation model

- the growth of working memory capacity over the course of parsing the corpus.

\subsection{Anaphora Resolution Results}

The results of the anaphora resolution algorithm are outlined in Figures 7.1 and 7.2 for the ACT-R model and Figures 7.3 and 7.4 for the Thompson model.

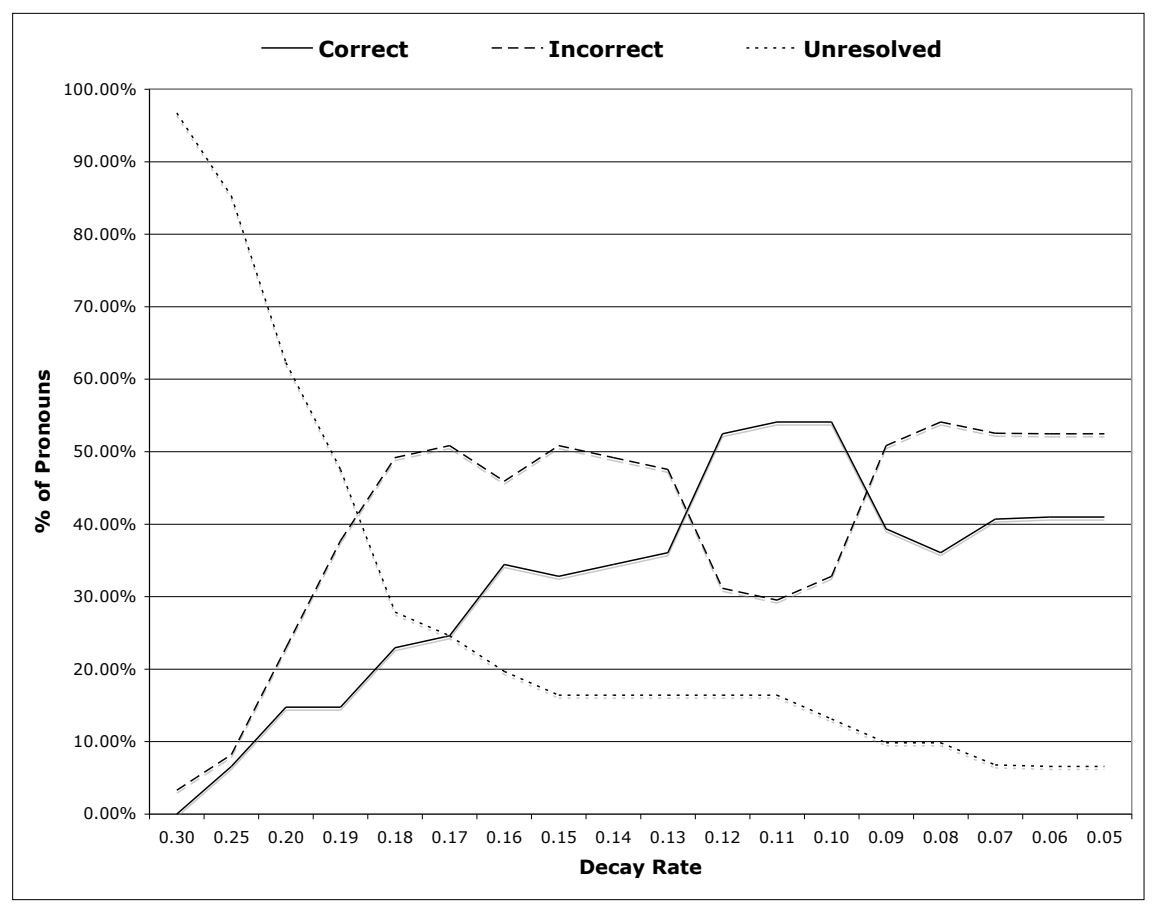

Figure 7.1: The ACT-R Model Resolution Results - 3 Brothers 


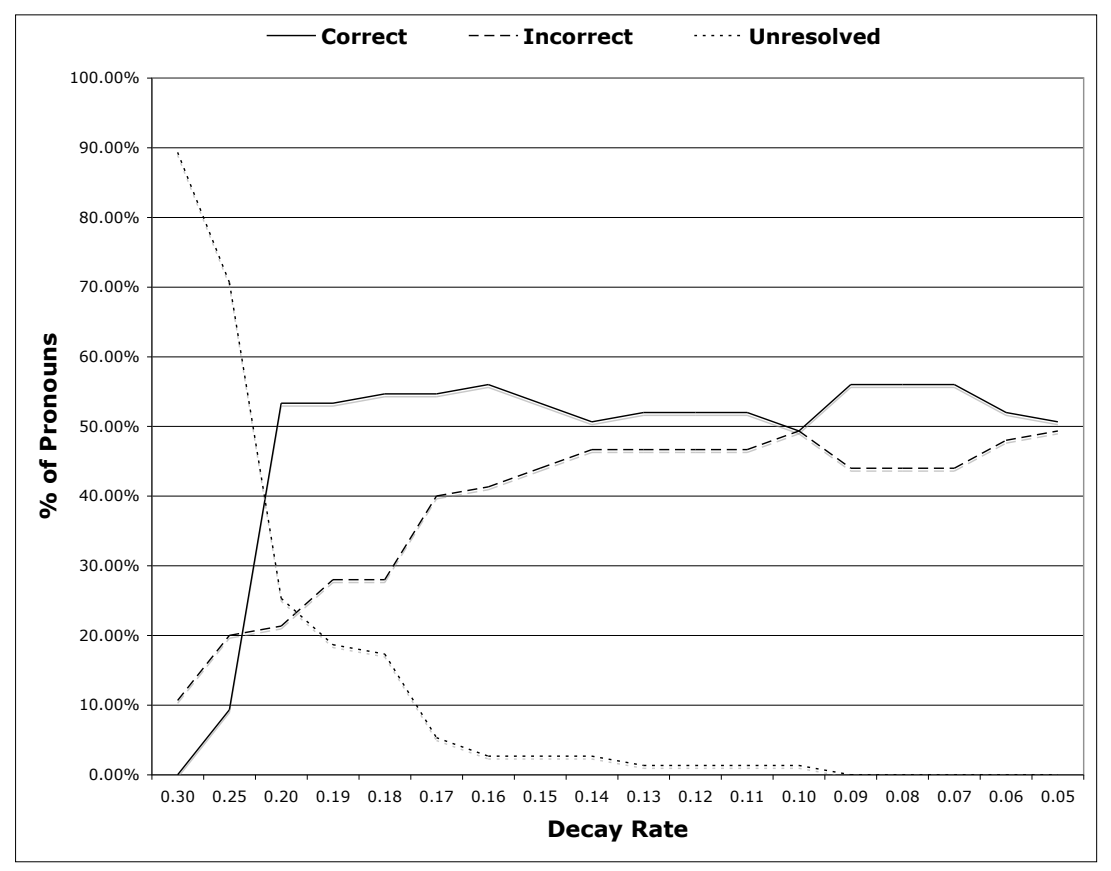

Figure 7.2: The ACT-R Model Resolution Results - Rumpelstiltskin

\subsection{Working Memory Capacity Results}

In addition to the results of the anaphora resolution algorithm, the behaviour of working memory was also observed for each decay rate. The working memory max capacity was the largest number of nouns that were observed to be in working memory at the end of each parsed sentence. Figures 7.5, 7.6, 7.7, and 7.8 outlined the maximal working memory contents for the ACT-R model and the Thompson model, respectively. A comparison of the growth rate of working memory capacity using the optimal decay rate is outlined in Figures 7.9 and 7.10.

\subsection{Discussion of Results}

A baseline comparison between the ACT-R model and the Thompson model can be made by considering the decay rates that give an equivalent, non-zero, number of unresolved pronouns. For The Three Brothers corpus, given the decay rate of 0.09, the ACT-R model 


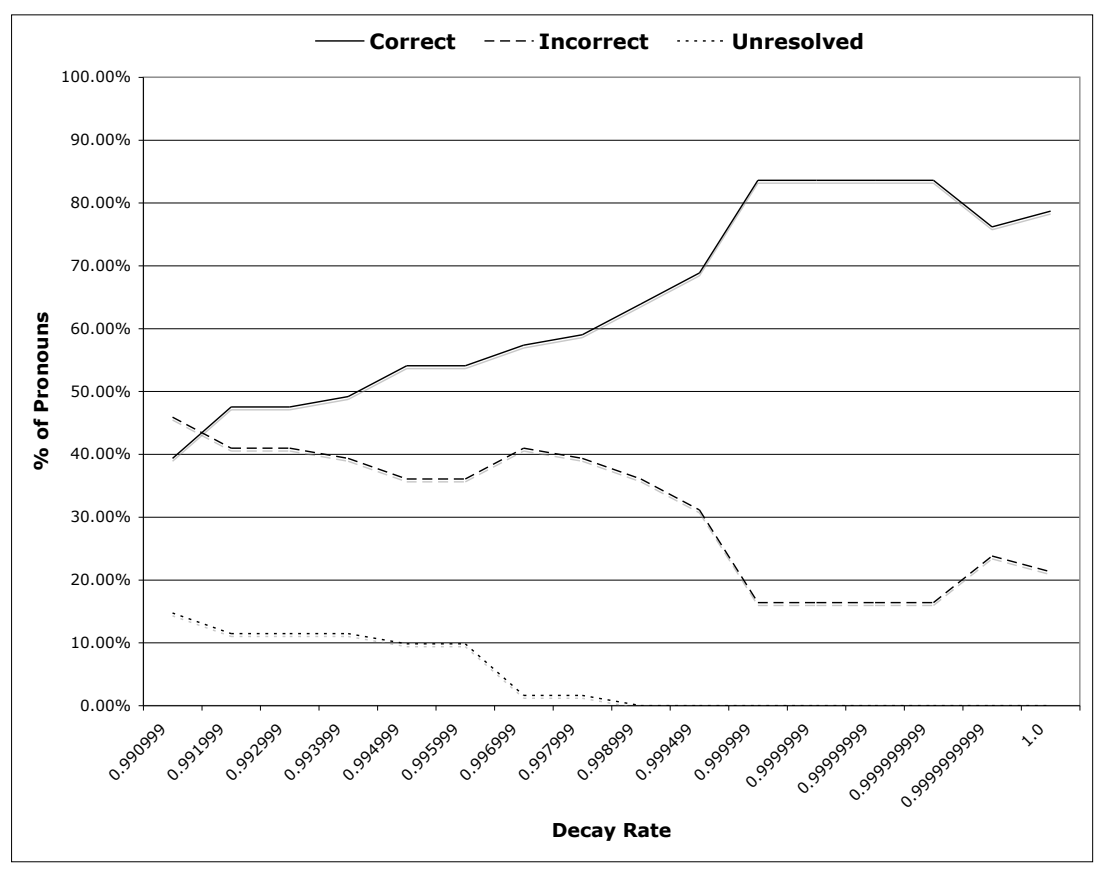

Figure 7.3: The Thompson Model Resolution Results - 3 Brothers

resolved $39.34 \%$ of the pronouns correctly. The Thompson model, given the decay rate of 0.995999, was able to resolved $54.10 \%$ of the pronouns correctly. Thus, in this comparison, the Thompson model achieved a higher success rate.

When the Rumpelstiltskin corpus is considered, the ACT-R model was able to correctly resolve $54.67 \%$ of the pronouns, given the decay rate of 0.17 . The Thompson model, given the decay rate of 0.995999 , was able to correctly resolve $42.67 \%$ of the pronouns. So, in this comparison, the ACT-R model achieved a higher success rate than the Thompson model.

When the overall range of results is examined, the Thompson model of activation achieved maximum success rates of $83.61 \%$ (The Three Brothers) and $86.67 \%$ (Rumpelstiltskin), while the ACT-R model of activation fell short with maximum success rates of $54.10 \%$ (The Three Brothers) and 56.00\% (Rumpelstiltskin). In general, the Thompson model was able to resolve a higher number of pronouns over a larger range of decay rates. Although the Thompson model achieved a higher overall success rate, a unresolved rate 


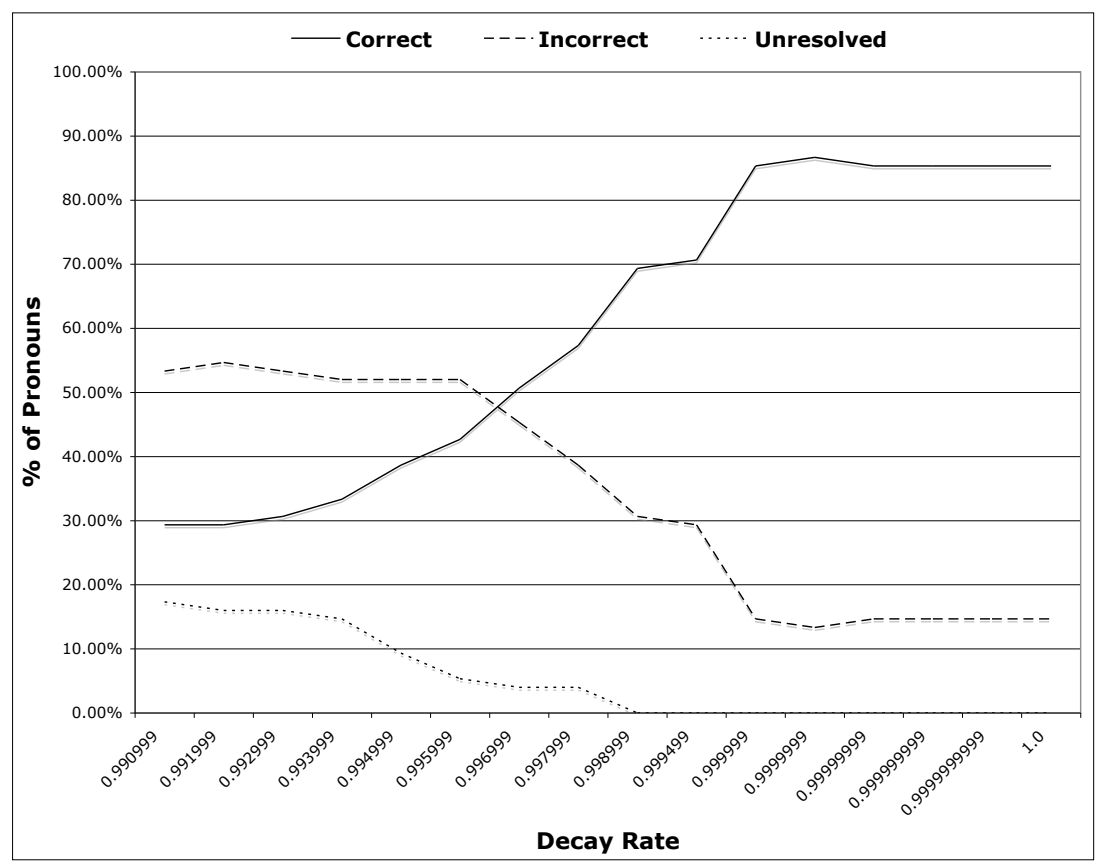

Figure 7.4: The Thompson Model Resolution Results - Rumpelstiltskin

of $0.0 \%$ is psychologically implausible based on the findings in [Levine et al 2000].

Examining Figures 7.9 and 7.10, it appears that the ACT-R model of activation had difficulty with concepts begin activated into working memory at the start of each corpus and then problems with getting those concepts out of working memory at the end of the corpus. This difficulty can be attributed to the fact that the ACT-R model of activation is based on the activation history. Concepts appear to move in and out of working memory more fluidly using the Thompson model of activation.

\subsection{Classification of Observed Error Types}

Throughout the testing of the implemented system, two types, or classes, of errors were observed. The first class of errors involved the incorrect resolution of a pronoun due to lack of information. The following sentence illustrates this error:

The son $_{1}$ that builds the best masterpiece will inherit his ${ }_{1}$ house. 


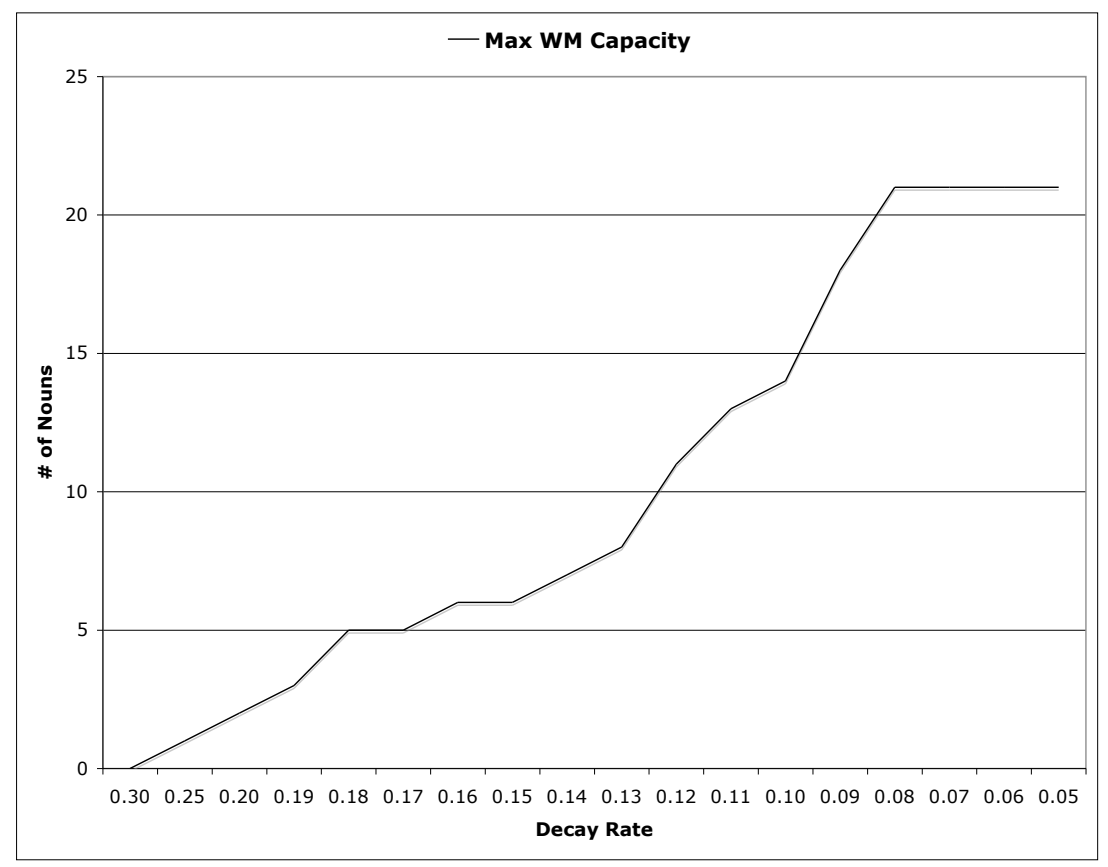

Figure 7.5: The ACT-R Model Working Memory Max Capacity - 3 Brothers

In this example, the system resolved the pronoun his with the noun son, which was incorrect. The pronoun actually references the noun father, which was mentioned earlier in the corpus. This incorrect resolution occurred because the noun son was the concept that had the highest antecedent score, and the accompanying noun house was not considered by the anaphora resolution algorithm. The second class of errors that was observed involved the incorrect resolution of pronouns that reference events. The next example illustrates this error:

$$
\text { The father } 1 \text { thought that this } 7 \text { was wonderful. }
$$

In this example, the pronoun this was incorrectly resolved to reference the noun horse from a previous sentence, which was not correct. The pronoun this actually references an event from earlier in the corpus. Since resolution of events was not within the scope of this thesis, occurrences of this type of error were not included as results. 


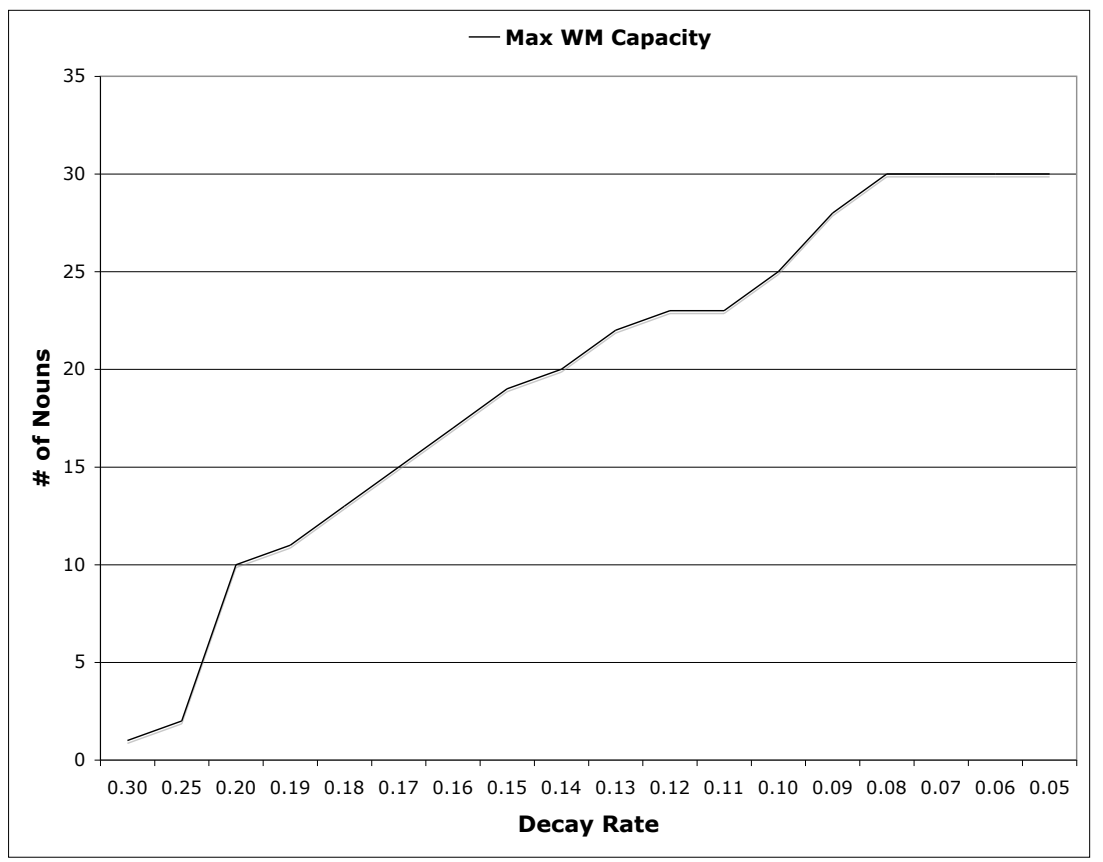

Figure 7.6: The ACT-R Model Working Memory Max Capacity - Rumpelstiltskin

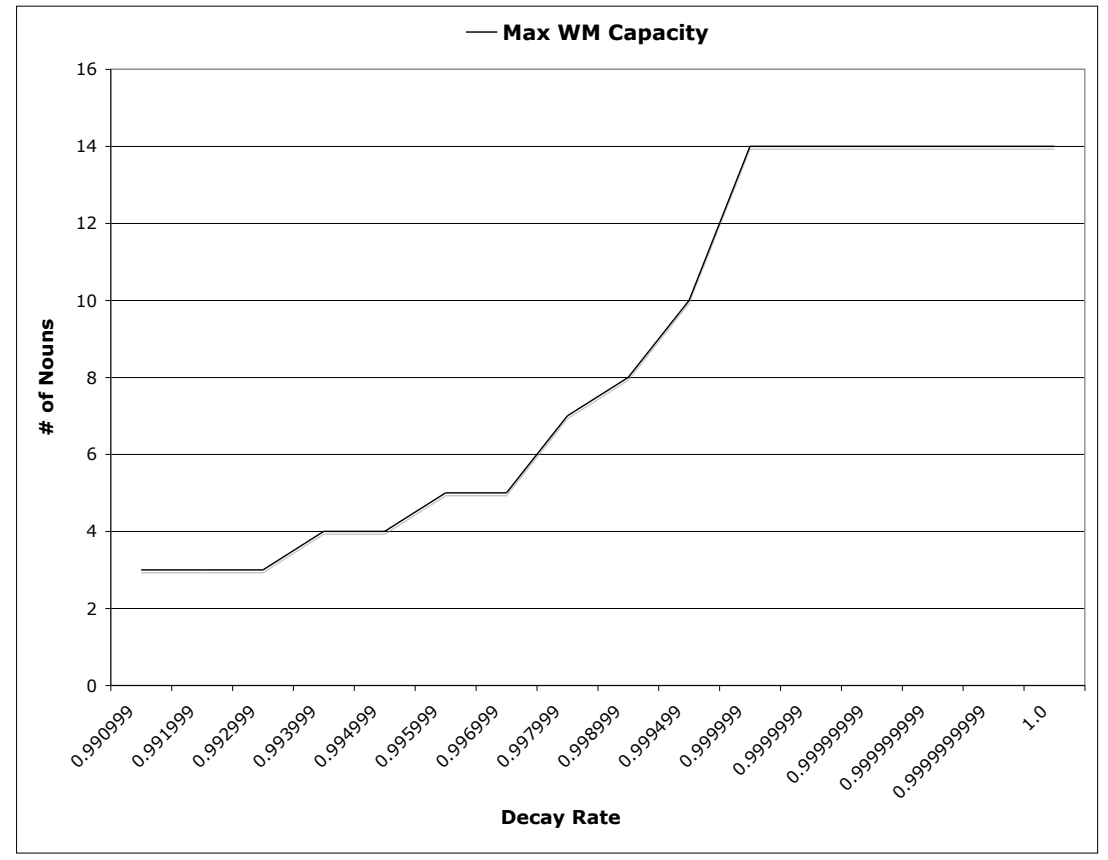

Figure 7.7: The Thompson Model Working Memory Max Capacity - 3 Brothers 


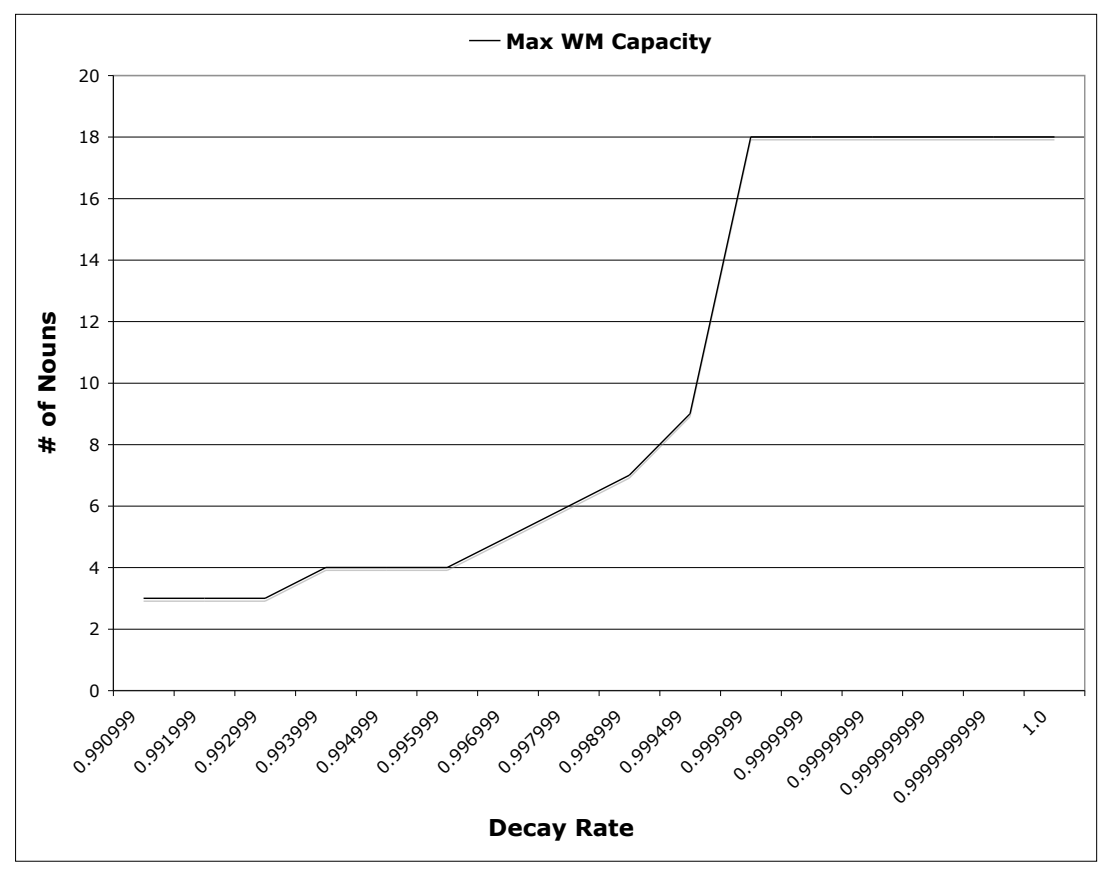

Figure 7.8: The Thompson Model Working Memory Max Capacity - Rumpelstiltskin

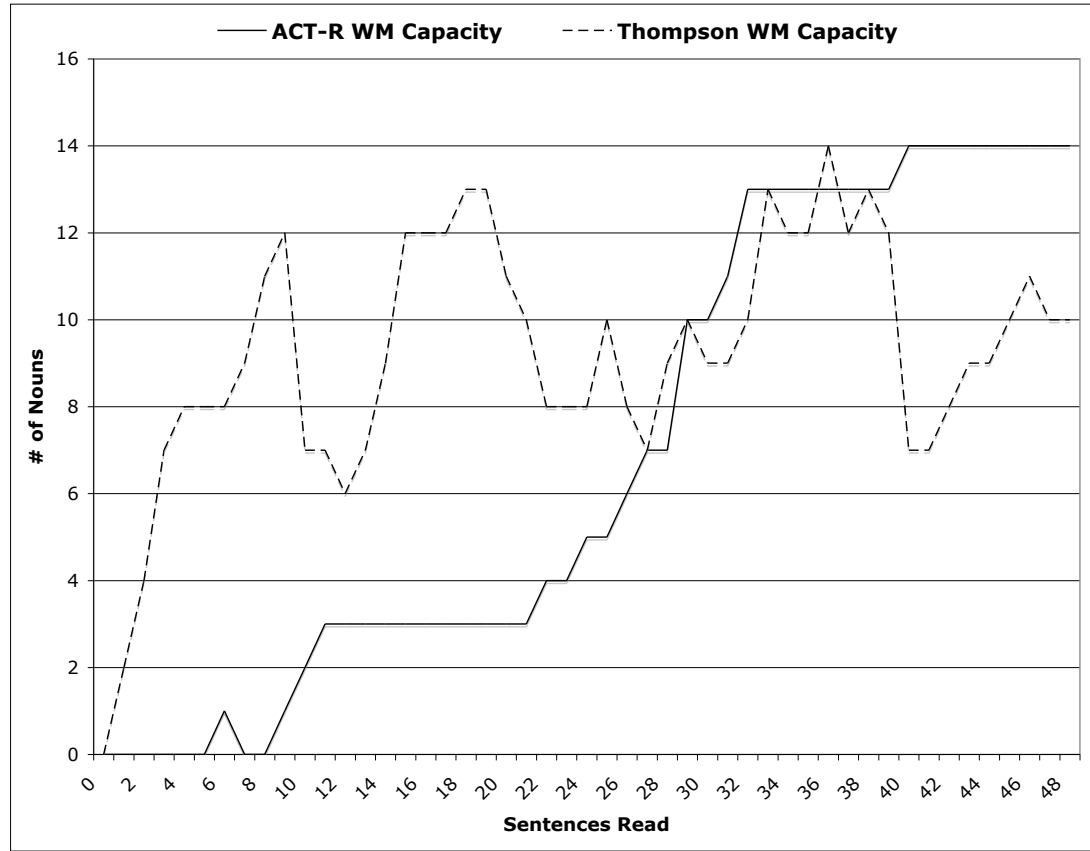

Figure 7.9: Working Memory Contents Comparison - 3 Brothers 


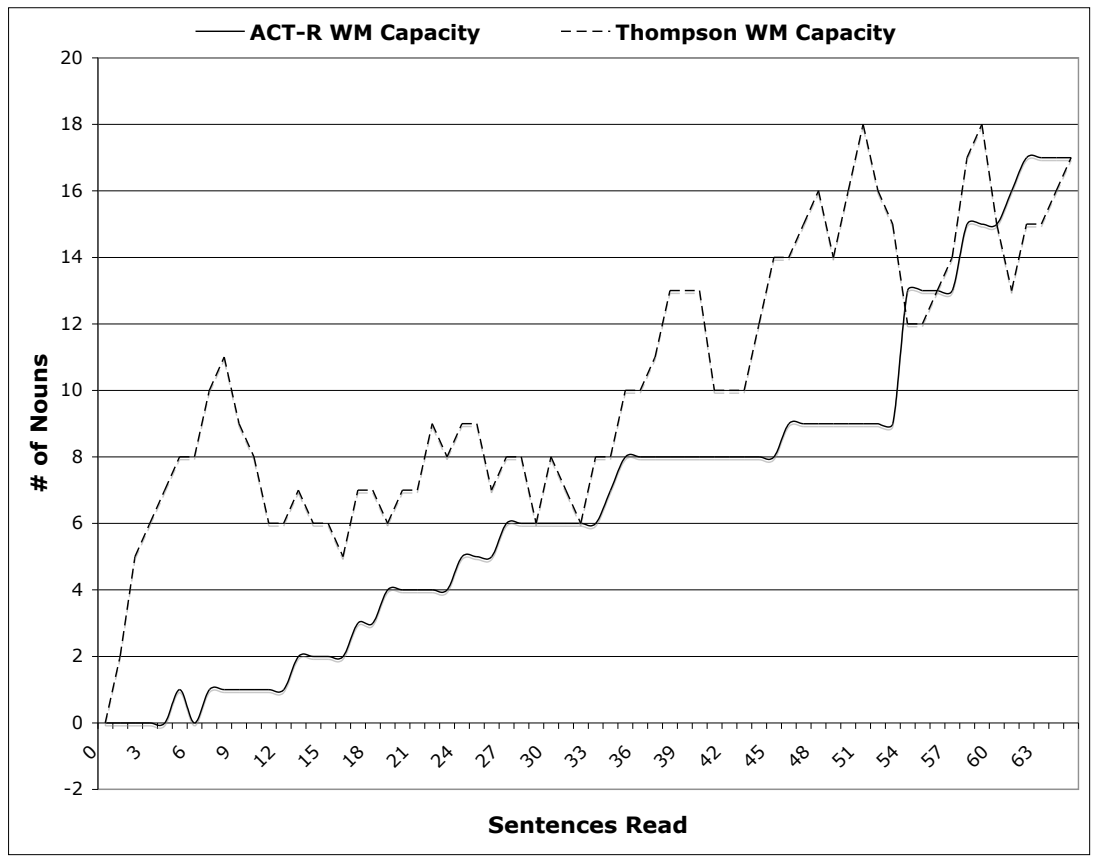

Figure 7.10: Working Memory Contents Comparison - Rumpelstiltskin 


\section{CHAPTER EIGHT}

\section{Conclusions and Future Work}

It will be happened; It shall be going to be happening; It will be was an event that could will have been taken place in the future.

- Arnold J. Rimmer (Future Echos)

\subsection{Comparison with Related Work}

\section{Anaphora Resolution}

Two of the biggest difficulties with making comparisons between anaphora resolution algorithms are the forthcomingness of authors to publish the results of their algorithms, and obtaining the corpora used for testing their algorithms. Over time, many corpora become difficult to obtain, thus making direct comparisons difficult. Early work such as [Sidner 1979], [Hirst 1981], and [Grosz and Sidner 1986], although popular in the fields of anaphora resolution and discourse analysis, fail to provide comprehensive results for their models. Table 8.1 shows the results obtained in this thesis compared to the work of other authors $^{1}$.

\section{Capacity of Working Memory}

Figures 7.9 and 7.10 illustrated the results of the dynamic capacity of working memory when an optimal ${ }^{2}$ decay rate was used. If a single concept is considered to be a chunk, the maximum capacity of the working memory model in this thesis appears to be much higher

\footnotetext{
${ }^{1}$ Mitkov, Callway, Hobbs, and Ferrández all give results for pronominal references. It is unknown whether these results included pronominal references to events or just nouns.

${ }^{2}$ Optimal decay rate was defined as the value that gave the highest anaphora resolution results.
} 


\begin{tabular}{|c|c|c|}
\hline Algorithm & Accuracy & Corpus \\
\hline \hline [Mitkov 1998] & $89.70 \%$ & Minolta Photocopier Manual and StyleWriter User's Guide \\
\hline [Hobbs 1986] & $91.70 \%$ & Early Civilization in China, Wheels, and Newsweek \\
\hline [Callaway and Lester 2002] & $97.80 \%$ & Little Red Riding Hood \\
\hline [Ferrández et al 1998] & $83.00 \%$ & TTU CCITT Handbook \\
\hline ACT-R & $55.05 \%$ & The Three Brothers and Rumpelstiltskin \\
\hline Thompson & $85.14 \%$ & The Three Brothers and Rumpelstiltskin \\
\hline
\end{tabular}

Table 8.1: Algorithm Comparison

than the capacity proposed in [Miller 1956], for short-term memory. These differences are possibly an artifact of the differences between what is considered a chunk in the human mind and what is considered a chunk in the model presented in this thesis.

\subsection{Future Work}

\section{Inclusion of Additional Semantic Structures}

Linking multiple semantic structures in the manner described in Chapter 4 could potentially increase the level of accuracy of anaphora resolution by providing additional contextual information. An excellent example is from The Three Brothers, where both the ACT-R model of activation and the Thompson model failed to resolve the antecedent for the sentence The son that builds the best masterpiece will inherit his house. Both models resolved the possessive pronoun his with the noun son, since son had the highest activation within working memory and highest feature set scoring. Unfortunately, this resolution is incorrect. The pronoun his should resolve to the noun father described in an earlier sentence. If an anaphora algorithm had information relating to the father-son relationship available, and information relating to the fact that the father owned a house, the algorithm could use this information to give a higher score to father, and resolve the antecedent correctly. Semantic representations, such as conceptual graphs, are effective in modelling this type of information. This modularization of modelling the behaviour of the human mind is supported by psychological literature. 


\section{Noun Instances in Working Memory and Long-Term Memory}

The models of long-term memory and working memory presented in this thesis only interact with single, generalized instances of nouns. For example, within the model of long-term memory, there exists only a single occurrence of the noun man. The existence of only one occurrence of a noun is problematic when defining the correctness of an anaphora resolution algorithm when a corpus contains multiple men. A possible solution to this problem is the introduction of an additional memory model that is a hybrid of working memory and long-term memory. Event memory, which was introduced in Chapter 3, is a model that could be adapted to handle nouns in addition to handling events. Event memory could contain instances of concepts that are generated as a corpus is read. The instance of a concept would gradually decay until only the most general concept exists. Long-term memory would act as a repository for generalized concepts, analogous to generalize event memory. The hierarchy of generalized concepts and instances of concepts could be realized by using the conceptual hierarchies introduced in Chapter 2 .

\section{Chart Parsing}

Chart parsing is a technique for bottom up parsing that avoids parsing the same structure more than once. Parsed sub-phrases are stored in a database called a chart, which is consulted when any type of backtracking occurs. A chart parser can be used to increase the speed of parsing while also creating a parser that is more tolerant to ungrammatical sentences [Allen 1995], [Russel and Norvig 1995], [Brown 2000], and [Thompson 2001a]. Potentially, a complete parse of a sentence would not be required for anaphora resolution. Parsing could be limited to just the noun phrase and verb phrase levels, and anaphora resolution could proceed from there. 


\subsection{Conclusion}

This thesis demonstrated the viability of using a modular, two-level memory model to perform anaphora resolution. Two models of human memory were used in conjunction with an anaphora resolution algorithm to solve the problem of pronominal references. Two models of concept activation and decay were implemented and subsequently tested on corpora of text with varying decay rates. The two-level memory model and anaphora resolution algorithm achieved resolution accuracy rates of up to 54.10\%(ACTR) and $83.61 \%$ (Thompson) for a modified version of The Three Brothers corpus, and $56.00 \%$ (ACT-R) and $86.67 \%$ (Thompson) for a modified version of the Rumpelstiltskin corpus. Although the results fall a bit short of the results from other works(with the exception of [Ferrández et al 1998]), these results are only a baseline for additional work. The model is intended to be an expansive model of human memory. It is theorized that adding additional semantic representations, and anaphora resolution algorithms, would increase the accuracy of the two-level memory model. 


\section{Bibliography}

[Allen 1995] James Allen. 1995. Natural Language Understanding. The Benjamin/Cummings Publishing Company, Redwood City, CA, 2nd edition. vii, 5, 11, 61

[Alshawi et al 1989] H. Alshawi, D.M. Carter, J. van Eijck, R.C. Moore, D.B. Moran, F.C.N. Pereira, S.G. Pulman, and A.G. Smith. 1989. Research Programme in Natural Language Processing. Technical report, SRI International, Cambridge, UK. vi, 13, 15, 45

[Altmann 1999] Gerry T.M. Altmann. 1999. Thematic Roles Assignment in Context. Journal of Memory and Language, 41(1):124-145, July. vi, 12, 13

[Anderson and Matessa 1997] John R. Anderson and Michael Matessa. 1997. A Production System Theory Of Serial Memory. Psychological Review, 104:728-748. 21, 22

[Anderson et al 1998] John R. Anderson, Dan Bothell, Christian Lebiere, and Michael Matessa. 1998. An Integrated Theory of List Memory. Journal of Memory and Language, 38:341-380, May. 22

[Anderson et al 2001] John R. Anderson, Raluca Budiu, and Lynne M. Reder. 2001. A Theory of Sentence Memory as Part of A General Theory of Memory. Journal of Memory and Language, 45:337-367, October. 20, 32

[Anderson 1976] John R. Anderson. 1976. Language, Memory, And Thought. Erlbaum, Hillsdale, NJ. 20

[Anderson 1983] John R. Anderson. 1983. The Architecture Of Cognition. Harvard University Press, Cambridge, MA. 20 
[Baddeley and Hitch 1974] Alan D. Baddeley and Graham Hitch, 1974. The psychology of learning and motivation: Advances in research and theory, volume 8, chapter Working Memory, pages 47-89. Academic Press, New York, NY. 18

[Baddeley 1986] Alan D. Baddeley. 1986. Working Memory. Oxford Psychology Series. Oxford University Press, Oxford. 19, 41

[Baddeley 1990] Alan D. Baddeley. 1990. Human Memory : Theory And Practice. Allyn Bacon, Boston. 19, 41

[Baddeley 2000] Alan Baddeley. 2000. The episodic buffer: a new component of working memory? Trends in Cognitive Sciences, 4(11):417-423, November. 18

[Belew 1987] R. K. Belew. 1987. A connectionist approach to conceptual information retrieval. In ICAIL '87: Proceedings of the 1st international conference on Artificial intelligence and law, pages 116-126, New York, NY, USA. ACM Press. 6

[Bennet 1995] Paul Bennet. 1995. A Course in Generalized Phrase Structure Grammar. Studies in Computational Linguistics. UCL Press Limited, London, England. 45

[Berger et al 2004] Helmut Berger, Michael Dittenbach, and Dieter Merkl. 2004. An adaptive information retrieval system based on associative networks. In CRPIT '04: Proceedings of the first Asian-Pacific conference on Conceptual modelling, pages 27-36, Darlinghurst, Australia, Australia. Australian Computer Society, Inc. 6

[Brown et al 2001] Charles Grant Brown, Lynda Williams, David Kubert, Clifford James Thompson, Neil Hagen, Dan Wolczuk, Cameron Rowe, Kurt Bolko, Jason Hess, Doug Smith, David Fidler, Mike Sluszkiewicz, John Everman, Richard Little, Ho Shum, and Alex Yung. 2001. The Language Engine Project 1996-2001. University of Northern British Columbia, Prince George, BC. 45

[Brown 2000] Charles Grant Brown. 2000. Natural Language Processing Manuscripts. University of Northern British Columbia, Prince George, BC. 61 
[Brown 2003] Charles Grant Brown. 2003. Lecture Notes in Computational Linguistics. University of Northern British Columbia, Prince George, BC. 34, 35, 46

[Bull 1960] W. Bull. 1960. Time, Tense, and the Verb: A Study in Theoretical and Applied Linguistics, with Particular Attention to Spanish. University of California Press, Berkeley, CA. 10

[Callaway and Lester 2002] Charles B. Callaway and James C. Lester. 2002. Pronominalization in General Discourse and Dialogue. In Proceedings of the 40th Annual Meeting on Association for Computational Linguistics, pages 88-95. Association for Computational Linguistics, July. 60

[Carter 1985] David Maclean Carter. 1985. A Shallow Approach To Anaphora Resolution. PhD Thesis 88, University Of Cambridge, Camdridge, England. 34, 35, 46

[Carter 1990] David M. Carter. 1990. Control Issues In Anaphora Resolution. Journel of Semantics, 7:435-454. 34, 35, 46

[Chung and Moldovan 1993] Sang-Hwa Chung and Dan I. Moldovan. 1993. Modeling Semantic Networks On The Connection Machine. Journal Of Parallel And Distributed Computing, 17:1993. 6

[Collins and Quillian 1969] A. Collins and M.R. Quillian. 1969. Retrieval Time from Semantic Memory. Journal of Verbal Leanring \& Verbal Behavior, 8:240-247. 6

[Culce-Murcia and Larsen-Freeman 1999] Marianne Culce-Murcia and Diane LarsenFreeman. 1999. The Grammar Book: An ESL/EFL Teacher's Course. Heinle \& Heinle, Boston, MA, 2nd edition. 10

[Federmeier and Kutas 1999] Kara D. Federmeier and Marta Kutas. 1999. A Rose by Any Other Name: Long-Term Memory Structure and Sentence Processing. Journal of Memory and Language, 41:469-495. 19 
[Ferrández et al 1998] A. Ferrández, M. Palomar, and L. Moreno. 1998. Anaphor resolution in unrestricted texts with partial parsing. In Proceedings of the 36th annual meeting on Association for Computational Linguistics, pages 385-391, Morristown, NJ, USA. Association for Computational Linguistics. 60, 62

[Foder 1983] Jerry A. Foder. 1983. Modularity of Mind: An Essay on Faculty Psychology. MIT Press, Cambridge, MA. 32

[Gathercole and Baddeley 1993] Susan E. Gathercole and Alan D. Baddeley. 1993. Working Memory and Language. Essays in Cognitive Psychology. Lawrence Erlbaum Associates, Hove, United Kingdom. 18, 41

[Geurts 1999] Paul Geurts. 1999. Presuppositions and Pronouns. Current Research in the Semantics/Pragmatics Interface. Elsevier, Amsterdam. 33

[Ginzburg and Cooper 2001] Jonathan Ginzburg and Robin Cooper. 2001. Resolving Ellipsis In Clarification. In Proceedings of 39th Annual Meeting of the Association for Computational Linguistics, pages 236-243. 33

[Grosz and Sidner 1986] Barbara J. Grosz and Candice L. Sidner. 1986. Attention, Intentions, and the Structure of Discourse. Computational Linguistics, 12(3):175-204. 34, 59

[Grosz 1977] Barbara J. Grosz. 1977. The Representation And Use Of Focus In Dialogue Understanding. Technical report, SRI International. 33, 34

[Group 2004] ACT-R Research Group. 2004. ACT-R Tutorial: Unit 1. http://actr.psy.cmu.edu/tutorials, January. 21

[Haarmann et al 2003] Henk J. Haarmann, Edddy J. Davelaar, and Marius Usher. 2003. Individual Differences In Semantic Short-Term Memory Capacity and Reading Comprehension. Journal of Memory and Language, 48:320-345. 19 
[Hardt 1997] Daniel Hardt. 1997. An Empirical Approach to VP Ellipsis. Computational Linguistics, 23(4):525-541, December. 33

[Hebb 1949] D.O. Hebb. 1949. The Organization of Behavior. Wiley, New York. 23, 27, 30

[Hirst 1981] Graeme Hirst. 1981. Discourse-oriented anaphora resolution in natural language understanding: a review. Computational Linguistics, 7(2):85-98. 59

[Hobbs 1986] Jerry R. Hobbs, 1986. Readings In Natural Language Processing, chapter Resolving Pronoun References, pages 339-352. Morgan Kaufmann Publishers, Los Altos, CA. 34, 35, 60

[Hunt 1973] Eart Hunt, 1973. Computer Models Of Thought And Language, chapter The Memory We Must Have, pages 343-371. Freeman and Company, San Francisco, CA. 22,23

[Kay 1971] P. Kay. 1971. Taxonomy and semantic contrast. Language, 47:866-887. 9

[Kazuhiro et al 1992] Kimura Kazuhiro, Suzuoka Takashi, and Amano Sin-ya. 1992. Association-based natural language processing with neural networks. In Proceedings of the 30th annual meeting on Association for Computational Linguistics, pages 224231, Morristown, NJ, USA. Association for Computational Linguistics. 6

[Koh and Clifton 2002] Sungryong Koh and Charles Clifton. 2002. Resolution of the Antecedent of a Plural Pronoun: Ontological Categories and Predicate Symmetry. Journal of Memory and Language, 46(4):830-844, May. 46

[Levine et al 2000] Willian H. Levine, Alexandria E. Guzmán, and Celia M. Klin. 2000. When Anaphor Resolution Fails. Journal of Memory and Language, 43(4):594-617, November. 37, 46, 54 
[Logie 1996] Robert H. Logie, 1996. The Seven Ages of Working Memory, pages 31-65. Counterpoints: Cognition, Memory, and Language. Oxford University Press, New York, NY. 17

[Luger and Stubblefield 1998] George F Luger and Wlliam A Stubblefield, 1998. Artificial Intelligence, chapter 14, pages 690-700. Addison Wesley Longman Inc, Reading, MA, 3rd edition. 27

[Ma and Isahara 2000] Qi Ma and Hitoshi Isahara. 2000. Semantic Networks Represented by Adaptive Associative Memories. Nerocomputing, 34:207-225, September. 6, 9

[McCulloch and Pitts 1943] W.S. McCulloch and W. Pitts. 1943. A logical calculas of the ideas immanent in nervous activity. Bulletin of Mathematical Biophysics, 5:115-133. 23

[Miller 1956] G.A. Miller. 1956. The Magical Number Seven, Plus or Minus Two: Some Limits On Our Capacity For Processing Information. Psychological Review, 63:81-97. $18,21,60$

[Mitkov 1998] Ruslan Mitkov. 1998. Robust pronoun resolution with limited knowledge. In Proceedings of the 36th annual meeting on Association for Computational Linguistics, pages 869-875, Morristown, NJ, USA. Association for Computational Linguistics. 34, 37,60

[Nash-Webber and Reiter 1977] Bonnie Nash-Webber and Raymond Reiter. 1977. Anaphora And Logical Form: On Formal Meaning Representations For Natural Language. In Proceedings of 5th International Joint Conference on Artificial Intelligence, volume 1, pages 121-131. 33

[Ockerbloom 2006] John Mark Ockerbloom. 2006. Grimm's Fairy Tales. http://www. cs.cmu.edu/ spok/grimmtmp. 49 
[Partee 1984] Barbara Partee. 1984. Nominal and Temporal Anaphora. Linguistics and Philosophy, 7:243-286. 33, 34

[Piwek and Krahmer 2000] Paul Piwek and Emiel Krahmer. 2000. Presuppositions in Context: Constructing Bridges. In Emiel Krahmer and Paul Piwek, editors, Varieties Of Anaphora, pages 87-96, Birmingham England. ESSLLI. 33

[Quillian 1968] M.A. Quillian, 1968. Semantic Information Processing, chapter Semantic Memory, pages 216-270. MIT Press, Cambridge, MA. 6

[Reichenbach 1947] H. Reichenbach. 1947. Elements of Symbolic Logic. Macmillian, New York. 11

[Richardson 1996] John T.E. Richardson, 1996. Evolving Concepts of Working Memory, chapter 1, pages 3-30. Counterpoints: Cognition, Memory, and Language. Oxford University Press, New York, NY. 17

[Rosch et al 1976] E. Rosch, C.B. Mervis, W.D. Gray, D.M. Johnson, and P. Boyes-Braem. 1976. Basic objects in natural categories. Cognitive Psychology, 8:382-439. 9

[Russel and Norvig 1995] Stuart J. Russel and Peter Norvig. 1995. Artificial Intelligence: A Modern Approach. Prentice Hall Series In Artificial Intelligence. Prentice Hall, Upper Saddle River, NJ. 24, 61

[Schank 1986] Roger C. Schank, 1986. Readings In Natural Language Processing, chapter Language And Memory, pages 171-191. Morgan Kaufmann Publishers Inc., Los Altos, CA. 22

[Sidner 1979] Candice L. Sidner. 1979. Toward A Computational Theory of Definite Anaphora Comprehension in English. PhD Thesis 537, MIT Artificial Intelligence Laboratory, Cambridge, MA. 34, 36, 59 
[Sidner 1983] Candice L. Sidner, 1983. Focusing in the Comprehension of Definite Anaphora, chapter 5, pages 267-330. M.I.T. Press, Cambridge, MA. 3, 34

[Sowa 1976] John F. Sowa. 1976. Conceptual Graphs for a Data Base Interface. IBM Journal of Research and Development, 20:336-357. 7

[Sowa 1979] John F. Sowa. 1979. Semantics of conceptual graphs. In Proceedings of the 17th annual meeting on Association for Computational Linguistics, pages 39-44, Morristown, NJ, USA. Association for Computational Linguistics. 7

[Sowa 1984] John F. Sowa. 1984. Conceptual Structures: Information Processing in Mind and Machine. Addison-Wesley, Reading, MA. 6, 7

[Sowa 2000] John F. Sowa. 2000. Knowledge Representation: Logical, Philosophical, and Computational Foundations. Brooks/Cole, Pacific Grove. 8, 12

[Sterling and Shapiro 1999] Leon Sterling and Ehud Shapiro. 1999. The Art of Prolog. MIT Press, 3rd edition. 44

[Thompson and Hagen 2001] Clifford James Thompson and Neil Hagen. 2001. CPSC 496 Relative Clauses, Quantifiers and Determiners. University of Northern British Columbia, Prince George, BC.

[Thompson 2001a] Clifford James Thompson. 2001a. CPSC 674 Bottom-Up Parsing: Chart Parsing. University of Northern British Columbia, Prince George, BC. 61

[Thompson 2001b] Clifford James Thompson. 2001b. CPSC 674 Grammars \& Syntactic Analysis. University of Northern British Columbia, Prince George, BC. 


\section{APPENDIX A}

\section{Corpora}

\section{The Three Brothers ${ }^{3}$}

There was a man who had three sons. He had nothing in the world. Each son wanted the house after his death. The Father loved them. He did not know what he should do. He did not wish to sell the house. It had belonged to his forefathers. He conceived a plan and told his sons that they must learn a trade. The son that builds the best masterpiece will inherit his house.

The sons were content with this. The first son was determined to be a blacksmith. The second son wanted to be a barber. The third son desired to be a fencing master. They set a time when they should come home.

The brothers found skillful masters who taught them their trades. The blacksmith had to shoe horses that belonged to the king. He believed that he would inherit the house. The barber shaved only distinguished people. He believed that his father would give the house to him. The fencing master suffered many blows to his body but he grit his teeth. He thought that he would win the house.

The brothers returned home to their father. They did not know when they would demonstrate their skills to their father. The brothers sat and contemplated what they could do. A hare ran across the field. The barber took his basin and soap. He lathered until the hare drew near. He soaped and saved the hare's whiskers while he was running at his top speed. He did not cut his skin or a hair on his body. The father was delighted.

A nobleman can in his coach and at full speed. The blacksmith ran towards the coach. He took four horseshoes off the horse while it was galloping and put new shoes on him. The father thought that this was wonderful.

\footnotetext{
${ }^{3}$ Adapted from http://www.cs.cmu.edu/ ${ }^{\text {spok/grimmtmp/094.txt }}$
} 
The third son asked to demonstrate his skills. It began to rain an the son drew sword. The sword flourished backwards and forwards above his head. No raindrops fell upon him. The rain fell harder and harder. He flourished his sword and remained dry. His father was amazed at this and gave his house to the third son.

His brothers were satisfied with this. They decided to line together since they loved eachother. The brothers continued their trades and earned a good living. They lived happily until they grew old. One brother became sick and died. The brothers grieved intensely and they became ill and died. They were laid in the same grave because they loved eachother. 


\section{Rumpelstiltskin ${ }^{4}$}

There was a miller who was poor but had a beautiful daughter. The miller visited the king and told him that his daughter could spin straw into gold. The king said that this was an art and pleased him. He requested that the miller bring his daughter to the palace.

The girl was brought to the king. He took her into a room that was filled with straw. She was given a spinning wheel and a reel. The king demanded that she complete the work by tomorrow or die. He locked the room and left the daughter. The poor daughter sat there and wept. She knew that she could not spin straw into gold.

The door opened and a little man entered the room. He asked the girl why she was crying. She told him that she must spin straw into gold. The little man told her that he could spin the straw for a price. He asked the girl what she could give to him. The daughter offered a necklace to him. The man took the necklace and sat at the spinning wheel. He spun the straw into gold.

The king returned in the morning and saw the gold. He was astonished and delighted. His heart filled with greed. The daughter was taken to a larger room that was filled with straw. He demanded that she complete the work by tomorrow.

The girl sat in the large room and cried. The little man returned and told her that he could spin the straw into gold. He asked her what she could give for the task. The daughter gave a ring to the small man. He grabbed the ring and spun the straw into gold.

The king returned and was amazed by the feat. He demanded that she spin more gold. The daughter was placed in a larger room. The king asked that she complete the task by the morning. He thought that she would be his wife when the task was completed. The manikin returned when the girl was alone. He asked what she would give for the task. She answered that she had nothing. The girl promised to give her first child when she becomes queen. She did not think that this would happen. The little man spun the straw into gold.

\footnotetext{
${ }^{4}$ Adapted from http://www.cs.cmu.edu/ ${ }^{\sim}$ spok/grimmtmp/044.txt
} 
The king returned in the morning. He found what he wished. The king took the girl in marriage and she became queen. She brought a beautiful child into the world. The queen gave no thought to the manikin. He entered her room and asked for her child. She was surprised and offered riches to him. The manikin refused the offer. The queen began to cry and the little man felt pity. He said that she could keep her child but she must guess his name in three days. She sent a messenger across the country. He searched for any name that might exist.

The manikin returned the next day. The queen guessed Casper and Melchior and Balthazar and other names that she knew. He said that she was incorrect. She sent a messenger on the second day. The queen asked for uncommon names. She guessed Shortribs and Sheepshanks and Laceleg but was incorrect.

The messenger found the manikin's house and overheard his name. The queen was delighted. The manikin returned on the final day and ask for a name. She guessed Conrad and Harry. He said that she was incorrect. She guessed Rumpelstiltskin. The manikin became angry and was pulled into the earth. 


\section{APPENDIX B}

\section{Tagged Corpora}

\section{The Three Brothers}

There was a $\operatorname{man}_{1}$ who had three $\operatorname{sons}_{2}$. $\mathrm{He}_{1}$ had nothing in the world. Each son wanted the house after his 1 death. The father ${ }_{1}$ loved them ${ }_{2}$. He ${ }_{1}$ did not know what he ${ }_{1}$ should do. $\mathrm{He}_{1}$ did not wish to sell the house $_{3}$. $\mathrm{It}_{3}$ had belonged to his forefathers. $\mathrm{He}_{1}$ conceived a plan and told his ${ }_{1}$ sons $_{2}$ that they 2 must learn a trade. The son $_{2 a b c}$ that builds the best masterpiece will inherit his ${ }_{1}$ house.

The $\operatorname{sons}_{2}$ were content with this. The first $\operatorname{son}_{2 a}$ was determined to be a blacksmith $2 a$. The second son $_{2 b}$ wanted to be a barber $2 b$. The third son $_{2 c}$ desired to be a fencing master $2 c$. They 2 set a time when they 2 should come home.

The brothers $_{2}$ found skillful masters $_{4}$ who taught them ${ }_{2}$ their 4 trades. The blacksmith $_{2 a}$ had to shoe horses that belonged to the king. $\mathrm{He}_{2 a}$ believed that he $2 a$ would inherit the house. The barber $2 b$ shaved only distinguished people. $\mathrm{He}_{2 b}$ believed that his $2 b$ father would give the house to him $2 b$. The fencing master $2 c$ suffered many blows to his $2 c$ body but he $2 c$ grit his $2 c$ teeth. $\mathrm{He}_{2 c}$ thought that he ${ }_{2 c}$ would win the house.

The brothers 2 returned home to their 2 father. They 2 did not know when they 2 would demonstrate their 2 skills to their ${ }_{2}$ father $_{1}$. The brothers ${ }_{2}$ sat and contemplated what they 2 could do. A hare 5 ran across the field. The barber $2 b$ took his $2 b$ basin and soap. $\mathrm{He}_{2 b}$ lathered until the hare 5 drew near. $\mathrm{He}_{2 b}$ soaped and saved the hare's $_{5}$ whiskers while he ${ }_{2 b}$ was running at his ${ }_{2 b}$ top speed. He ${ }_{2 b}$ did not cut his 5 skin or a hair on his 5 body. The father 1 was delighted.

A nobleman 6 can in his 6 coach and at full speed. The blacksmith $2 a$ ran towards the coach. $\mathrm{He}_{2 a}$ took four horseshoes off the horse ${ }_{7}$ while it 7 was galloping and put new shoes on him 7 . The father ${ }_{1}$ thought that this was wonderful. 
The third son $2 c$ asked to demonstrate his $2 c$ skills. It began to rain and the son $2 c$ drew his $2 c$ sword. The sword flourished backwards and forwards above his $2 c$ head. No raindrops fell upon $\operatorname{him}_{2 c}$. The rain fell harder and harder. $\mathrm{He}_{2 c}$ flourished his $2 c$ sword and remained dry. His ${ }_{2 c}$ father ${ }_{1}$ was amazed at this and gave his 1 house ${ }_{3}$ to the third son $2 c$.

His $_{2 c}$ brothers 2 were satisfied with this. They 2 decided to line together since they 2 loved eachother. The brothers ${ }_{2}$ continued their 2 trades and earned a good living. They 2 lived happily until they 2 grew old. One brother became sick and died. The brothers 2 grieved intensely and they 2 became ill and died. They 2 were laid in the same grave because they 2 loved eachother. 


\section{Rumpelstiltskin}

There was a miller 1 who was poor but had a beautiful daughter 2 . The miller 1 visited the king $_{3}$ and told him $_{3}$ that his ${ }_{1}$ daughter $_{2}$ could spin straw into gold. The king 3 said that this was an art and pleased him ${ }_{3}$. $\mathrm{He}_{3}$ requested that the miller ${ }_{1}$ bring his daughter 2 to the palace.

The girl ${ }_{2}$ was brought to the $\mathrm{ling}_{3}$. $\mathrm{He}_{3}$ took $\mathrm{her}_{2}$ into a room that was filled with straw. She 2 was given a spinning wheel and a reel. The king ${ }_{3}$ demanded that she ${ }_{2}$ complete the work by tomorrow or die. $\mathrm{He}_{3}$ locked the ${ }_{\text {room }} 6$ and left the daughter 2 . The poor daughter $_{2}$ sat there 6 and wept. She 2 knew that she 2 could not spin straw into gold.

The door opened and a little $\operatorname{man}_{4}$ entered the room. $\mathrm{He}_{4}$ asked the girl 2 why she $_{2}$ was crying. She 2 told him him $_{4}$ that she $_{2}$ must spin straw into gold. The little $\operatorname{man}_{4}$ told her $_{2}$ that he 4 could spin the straw for a price. He $_{4}$ asked the girl 2 what $\operatorname{she}_{2}$ could give to him 4 . The daughter $_{2}$ offered a necklace to him ${ }_{4}$. The $\operatorname{man}_{4}$ took the necklace and sat at the spinning wheel. $\mathrm{He}_{4}$ spun the straw into gold.

The king 3 returned in the morning and saw the gold. $\mathrm{He}_{3}$ was astonished and delighted. His 3 heart filled with greed. The daughter 2 was taken to a larger room that was filled with straw. $\mathrm{He}_{3}$ demanded that $\mathrm{she}_{2}$ complete the work by tomorrow.

The girl 2 sat in the large room and cried. The little $\operatorname{man}_{4}$ returned and told her ${ }_{2}$ that he ${ }_{4}$ could spin the straw into gold. $\mathrm{He}_{4}$ asked her ${ }_{2}$ what she ${ }_{2}$ could give for the task. The daughter ${ }_{2}$ gave a ring to the small $\operatorname{man}_{4}$. He $\mathrm{H}_{4}$ grabbed the ring and spun the straw into gold.

The $\mathrm{king}_{3}$ returned and was amazed by the feat. $\mathrm{He}_{3}$ demanded that $\mathrm{she}_{2}$ spin more gold. The daughter 2 was placed in the largest room. The king 3 asked that she ${ }_{2}$ complete the task by the morning. $\mathrm{He}_{3}$ thought that she ${ }_{2}$ would be his ${ }_{3}$ wife $_{2}$ when the task was completed. The manikin 4 returned when the girl 2 was alone. $\mathrm{He}_{4}$ asked what she 2 would give for the task. She ${ }_{2}$ answered that she ${ }_{2}$ had nothing. The girl 2 promised to give her ${ }_{2}$ first child when she $_{2}$ becomes queen $_{2}$. She ${ }_{2}$ did not think that 
this would happen. The little man 4 spun the straw into gold.

The king 3 returned in the morning. $\mathrm{He}_{3}$ found what he ${ }_{3}$ wished. The king 3 took the $\operatorname{girl}_{2}$ in marriage and $\operatorname{she}_{2}$ became queen $_{2}$. She ${ }_{2}$ brought a beautiful child into the world. The $\overline{\text { queen }}_{2}$ gave no thought to the manikin ${ }_{4}$. $\mathrm{He}_{4}$ entered her ${ }_{2}$ room and asked for $\operatorname{her}_{2}$ child. She 2 was surprised and offered riches to him ${ }_{4}$. The manikin 4 refused the offer. The queen $_{2}$ began to cry and the little $\operatorname{man}_{4}$ felt pity. $\mathrm{He}_{4}$ said that she $_{2}$ could keep her 2 child but she $_{2}$ must guess his ${ }_{4}$ name in three days. She ${ }_{2}$ sent a messenger 5 across the country. He 5 searched for any name that might exist.

The manikin 4 returned the next day. The queen 2 guessed Casper and Melchior and Balthazar and other names that she knew. $\mathrm{He}_{4}$ said that she ${ }_{2}$ was incorrect. She 2 sent a messenger $_{5}$ on the second day. The queen $_{2}$ asked for uncommon names. She 2 guessed Shortribs and Sheepshanks and Laceleg but was incorrect.

The messenger 5 found the manikin's ${ }_{4}$ house and overheard his ${ }_{4}$ name. The queen 2 was delighted. The manikin 4 returned on the final day and ask for a name. She 2 guessed Conrad and Harry. $\mathrm{He}_{4}$ said that $\mathrm{she}_{2}$ was incorrect. She ${ }_{2}$ guessed Rumpelstiltskin. The manikin 4 became angry and was pulled into the earth. 